\title{
A Homogenized Energy Model for Hysteresis in Ferroelectric Materials: General Density Formulation
}

\author{
Ralph C. Smith and Andrew Hatch \\ Department of Mathematics \\ Center for Research in Scientific Computation \\ North Carolina State University \\ Raleigh, NC 27695 \\ rsmith@eos.ncsu.edu, aghatch@eos.ncsu.edu
}

\author{
Binu Mukherjee and Shifang Liu \\ Department of Physics \\ Royal Military College of Canada \\ Kingston, Ontario, Canada K7K 7B4 \\ mukherjee@rmc.ca, liu-s@rmc.ca
}

\begin{abstract}
In this paper, we construct a framework for modeling hysteresis and constitutive nonlinearities in ferroelectric compounds based on energy analysis at mesoscopic scales in combination with stochastic homogenization techniques to construct macroscopic models. In the first step of the development, previous analysis is used to construct Helmholtz and Gibbs energy relations at the lattice level. This provides local polarization relations which can be extrapolated to provide constitutive models for certain homogeneous, single crystal compounds. To incorporate material and field nonhomogeneities, as well as the effects of polycrystallinity, certain parameters in the local models are assumed to be manifestations of underlying distributions having densities which must be identified for a given compound. Two techniques for estimating the unknown densities are presented, and the accuracy of the resulting model is illustrated for both symmetric major loops and biased minor loops through fits and predictions with experimental PZT4 and PZT5H data.
\end{abstract}




\section{Introduction}

The use of ferroelectric compounds as actuators and sensors in high performance control applications has burgeoned in recent years due to the high set point accuracy and broadband capabilities of the materials. For example, piezoceramic rods or shells are employed as positioning mechanisms in all present atomic force microscope (AFM) and scanning tunneling microscope (STM) designs to achieve the nanoscale tolerances required by the devices $[8,26,27]$ whereas relaxor ferroelectrics have received significant attention as potential sonar transducers [7, 11] due to the large power density to weight ratios exhibited by the materials. However, these advantages are accompanied by several nonlinear material attributes - hysteresis is present in the piezoceramic materials at all drive levels as shown in Figure 1 whereas relaxor ferroelectric compounds exhibit constitutive nonlinearities and significant temperature-dependency for high drive level operation in anhysteretic regimes. These nonlinear mechanisms must be accommodated in some manner to achieve the novel control capabilities provided by these materials in high performance applications.

In many cases, feedback control loops can be designed to minimize nonlinear effects and this has led to the successful use of piezoceramic and relaxor ferroelectric transducers for a broad range of applications. However, this technique can be difficult to implement at moderate to high frequencies where increasing noise-to-signal ratios and diminishing characteristics of high pass filters reduce the effectiveness of control designs based solely on linear feedback laws. The use of charge or current controlled amplifiers can also mitigate the majority of hysteresis and constitutive nonlinearities exhibited by ferroelectric materials [17, 18]. This can be expensive, however, when compared with the more common voltage-controlled amplifiers, and current-controlled amplifiers cannot maintain DC offsets as required by numerous applications - e.g., the $x$-state in an AFM must hold a fixed position during sweeps in the $y$-stage. This motivates the development of model-based frameworks which are sufficiently accurate to quantify field, temperature, stress, and rate-dependent attributes of ferroelectric materials and sufficiently efficient to permit real-time implementation.

The PZT5H data, plotted in Figure 1, illustrates a number of the attributes which comprehensive models must encompass for low frequency, fixed-temperature, fixed-stress regimes. At low field levels, the materials exhibit a quadratic Raleigh loop behavior whereas they exhibit saturation nonlinearities and significant hysteresis at large field levels. In quasistatic and low frequency regimes, models must

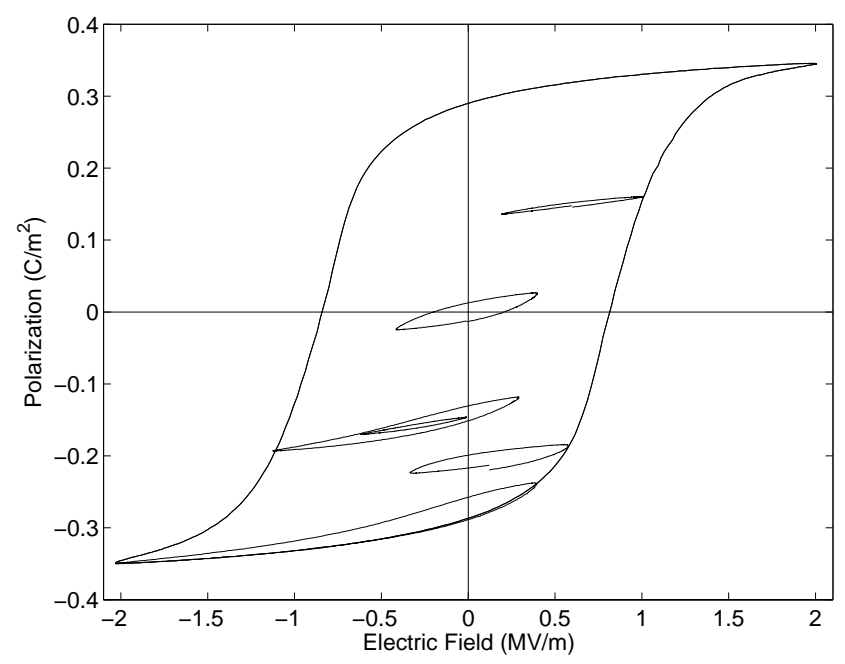

Figure 1: Quasistatic PZT5H data collected at $0.2 \mathrm{~Hz}$ including a symmetric major loop, a symmetric Rayleigh loop, and biased minor loops. 
guarantee closure of biased minor loops and enforce the 'deletion' or 'wiping out' property as minor loops are exited while including mechanisms to address accommodation and after-effects (relation phenomena). Additionally, the materials exhibit stress, frequency and temperature-dependencies which we address only peripherally in subsequent discussion.

As detailed in [30, 31, 32], there exist a broad range of techniques for modeling hysteresis in ferroelectric compounds including microscopic models [23] and both energy-based and purely phenomenological macroscopic models. We focus on the latter category to accommodate the emphasis on transducer and control design.

Representative examples of energy-based modeling frameworks are the domain wall theory presented in [30,31], the theory of Chen and Lynch [4] and Huang and Tiersten [12], and the homogenized free energy theory [32]. All of these approaches have both strengths and weaknesses depending upon the operating regimes. For example, the domain wall models are highly efficient for quantifying symmetric major loops but require significant extension to guarantee minor loop closure without $a$ priori knowledge of turning points which, unfortunately, will be the case in feedback control applications. The purely phenomenological models include Preisach models which were developed in the context of magnetic materials [24] and have been widely employed for a vast range of applications $[2,3]$ including ferroelectric materials $[10,25]$. The advantage of the Preisach approach lies in its generality and rigorous mathematical foundation. It has the disadvantage that this generality often precludes the use of physical measurements to identify parameters or update parameters to accommodate changing operating conditions. Furthermore, the classical Preisach theory must be modified in the manner described in [9] for magnetic materials to accommodate measured reversible effects, noncongruency, and temperature, stress and rate-dependencies.

The theory presented here combines aspects of the homogenized free energy theory of [32] and Preisach models posed in terms of general densities or measures. In the theory of [32], Helmholtz and Gibbs energy relations are constructed at the lattice, or mesoscopic, level and Boltzmann theory is employed to construct local polarization relations. To construct macroscopic models, it is assumed that local coercive and effective fields are manifestations of underlying lognormal and normal densities. Stochastic homogenization in this manner provides constitutive models for the $E$ - $P$ relation which quantify hysteresis and certain material nonlinearities exhibited by ferroelectric materials. However, the models have limited accuracy for certain materials due to the restrictions imposed through the a priori assumption of lognormal and normal densities.

In this paper, we reformulate the modeling framework in terms of general densities to be identified through a fit to measured data from a given compound. We also present three identification strategies and illustrate the performance of the model through comparison and prediction of PZT4 and PZT5H data.

The formulation of Preisach models in terms of stochastically homogenized free energy models was first proposed in [33] and the proposed framework extends this analysis. The construction of hysterons or kernels from energy principles has the advantage that it includes reversible effects directly and incorporates certain temperature and rate effects in the basis rather than in the densities or parameters as is the case for classical Preisach models. Stochastic homogenization through formulation in terms of general densities, as motivated by Preisach models, provides models with significant accuracy and flexibility. The resulting models guarantee the closure of biased minor loops in quasistatic or low frequency conditions but include mechanisms which characterize accommodation (reptation) and after-effect (relaxation) phenomena. Hence the framework incorporates a number of the phenomena exhibited by ferroelectric materials while providing the efficiency required for eventual implementation.

From the perspective of model-based control design, construction in terms of general densities provides the important advantage that models exhibit a linear dependence on the parameters as 
compared with the nonlinear dependence which results from the a priori choice of lognormal and normal densities. This permits consideration of a wide range of linear adaptive identification and control strategies in the former case whereas nonlinear adaptive identification strategies of the type developed in [22] are required in the latter case and few options exist for adaptive control.

\section{Free Energy Model for Polycrystalline Compounds}

We summarize here the hysteresis models developed in [32] for nonhomogeneous, polycrystalline ferroelectric compounds. In the first step of the model development, Helmholtz and Gibbs energy relations are constructed at the lattice level to quantify the balance between the internal and electrostatic energies. In the absence of thermally induced relaxation mechanisms, minima of the Gibbs relations provides a mesoscopic polarization model whereas a balance of the thermal and Gibbs energy through Boltzmann principles is employed for regimes in which relaxation or rate-dependent phenomena are significant. For homogeneous, single crystal compounds, the mesoscopic models derived through either set of assumptions can be applied throughout the material to provide a macroscopic model quantifying the hysteresis and constitutive nonlinearities inherent to ferroelectric materials. For nonhomogeneous, polycrystalline materials, variations in the lattice are incorporated by assuming that certain parameters in the mesoscopic Gibbs relations are manifestations of underlying distributions rather than constant values. In the models derived in [32] and summarized in this section, we make the a priori assumption that the local coercive fields $E_{c}$ are distributed with a lognormal density whereas effective fields are assumed to be normally distributed about the applied field $E$. The first assumption enforces the positivity of $E_{c}$ whereas the second is based on the tenet that local dipole interactions satisfy the central limit theorem. As illustrated in the examples of Section 4 and [32], the resulting macroscopic models accurately quantify the hysteresis and constitutive nonlinearities for PZT5A and PZT5H in symmetric drive regimes but have limited accuracy for hard PZT4 compounds or drive regimes yielding multiple, biased minor loops.

In Section 3, we relax the assumption that $E_{c}$ and $E_{i}$ have lognormal and normal densities and consider instead the formulation of the model in terms of general densities $\nu$ to be identified through a fit to data for a given material. While this requires additional complexity when constructing the model, it yields superior accuracy when characterizing general ferroelectric compounds in complex drive regimes.

\subsection{Mesoscopic Polarization Models}

For fixed temperature regimes, it is illustrated in [32] that an appropriate construct for the Helmholtz energy at the lattice level is

$$
\psi(P)= \begin{cases}\frac{1}{2} \eta\left(P+P_{R}\right)^{2} & , P \leq-P_{I} \\ \frac{1}{2} \eta\left(P-P_{R}\right)^{2} & , P \geq P_{I} \\ \frac{1}{2} \eta\left(P_{I}-P_{R}\right)\left(\frac{P^{2}}{P_{I}}-P_{R}\right) & ,|P|<P_{I} .\end{cases}
$$

Here $P_{I}$ and $P_{R}$ respectively denote the inflection point in the $C_{1}$ energy relation and the positive point at which a minimum of $\psi$ occurs as depicted in Figure 2. The relation (1) quantifies the internal energy at the lattice level in the absence of applied fields.

To incorporate the work due to applied fields $E$, we also consider the Gibbs energy relation

$$
G(E, P)=\psi(P)-E P
$$



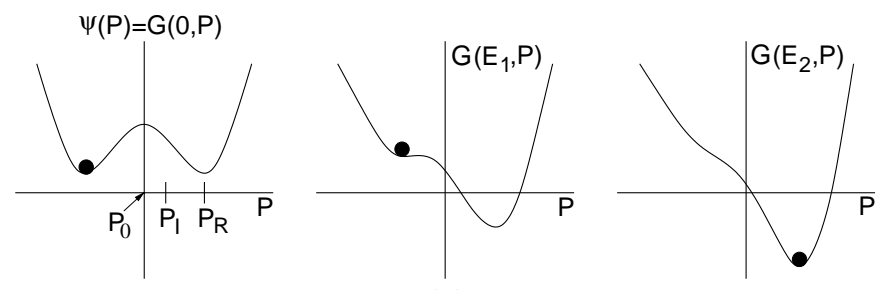

(a)
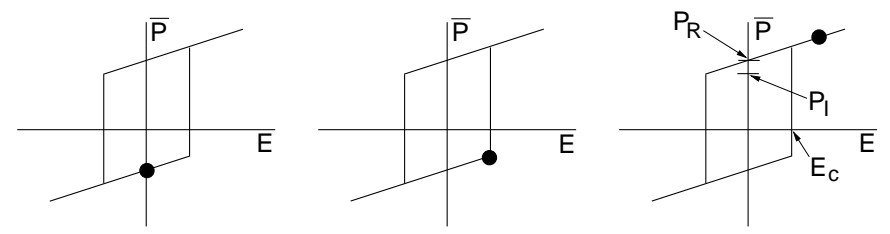

(b)

Figure 2: Helmholtz energy $\psi$ and Gibbs energy $G$ for increasing field $E\left(E_{2}>E_{1}>0\right)$. (b) Dependence of the local average magnetization $\bar{P}$ on the field in the absence of thermal activation.

where the latter term incorporates the potential energy $U=-\mathbf{p} \cdot \mathbf{E}$ of a dipole $\mathbf{p}$ in a uniaxial field. In the absence of an applied stress $\sigma$, the Gibbs relation (2) characterizes the energy landscape in homogeneous, polycrystalline materials.

To quantify the local average polarization $\bar{P}$ depicted in Figure 2(b), we consider two cases: (i) drive regimes in which relaxation mechanisms are negligible, and (ii) regimes in which thermallyinduced relaxation is sufficiently prominent to warrant inclusion in the model.

In the absence of thermal relaxation, the necessary conditions $\frac{\partial G}{\partial P}=0, \frac{\partial^{2} G}{\partial P^{2}}>0$ are invoked to yield the general relation

$$
\bar{P}=\frac{1}{\eta} E+\delta P_{R}
$$

for the local average polarization. Here $\delta=1$ for positively oriented dipoles and $\delta=-1$ for negative orientations. The expression (3) illustrates that $P_{R}$ can be interpreted as a local or latticelevel remanence value whereas $\eta$ is the reciprocal slope $\frac{\partial E}{\partial P}$ after switching. To quantify the dipole orientations formally indicated by $\delta$, we employ the Preisach notation (e.g., see [2])

$$
\left[\bar{P}\left(E ; E_{c}, \xi\right)\right](t)= \begin{cases}{\left[\bar{P}\left(E ; E_{c}, \xi\right)\right](0)} & , \tau(t)=\emptyset \\ \frac{E}{\eta}-P_{R} & , \tau(t) \neq \emptyset \text { and } E(\max \tau(t))=-E_{c} \\ \frac{E}{\eta}+P_{R} & , \tau(t) \neq \emptyset \text { and } E(\max \tau(t))=E_{c} .\end{cases}
$$

Here

$$
\left[\bar{P}\left(E ; E_{c}, \xi\right)\right](0)= \begin{cases}\frac{E}{\eta}-P_{R} & , E(0) \leq-E_{c} \\ \xi & ,-E_{c}<E(0)<E_{c} \\ \frac{E}{\eta}+P_{R} & , E(0) \geq E_{c}\end{cases}
$$

denotes the initial dipole distribution and transition times are designated by

$$
\tau(t)=\left\{t \in\left(0, T_{f}\right] \mid E(t)=-E_{c} \text { or } E(t)=E_{c}\right\}
$$

where $T_{f}$ denotes the final time under consideration. The dependence of the kernel $\bar{P}$ on the local coercive field $E_{c}$ is indicated as a prelude to the discussion in Section 2.2 where $E_{c}$ is assumed to be distributed. 
For operating regimes in which relaxation mechanisms are significant, it is necessary to balance the Gibbs energy $G$ with the relative thermal energy $k T / V$ - where $k$ denotes Boltzmann's constant - through the Boltzmann relation

$$
\mu(G)=C e^{-G V / k T} .
$$

It is observed that the probability $\mu$ of obtaining an energy level $G$ is increased when the magnitude of $k T / V$ approaches that of $G$. The constant $C$ is chosen to ensure a probability of one for integration over all possible dipole orientations.

If we let $x_{+}$and $x_{-}$denote the fractions of dipoles having positive and negative orientations, and let $\left\langle P_{+}\right\rangle$and $\left\langle P_{-}\right\rangle$denote the average expected polarizations associated with the two orientations, the local average polarization at the lattice level is

$$
\bar{P}=x_{+}\left\langle P_{+}\right\rangle+x_{-}\left\langle P_{-}\right\rangle .
$$

Since the expected polarization values are obtained by integrating the product $P \mu(G(P))$ over all admissible configurations, it follows that

$$
\left\langle P_{+}\right\rangle=\frac{\int_{P_{I}}^{\infty} P e^{-G(E, P, T) V / k T} d P}{\int_{P_{I}}^{\infty} e^{-G(E, P, T) V / k T} d P} \quad, \quad\left\langle P_{-}\right\rangle=\frac{\int_{-\infty}^{-P_{I}} P e^{-G(E, P) V / k T} d P}{\int_{-\infty}^{-P_{I}} e^{-G(E, P) V / k T} d P} .
$$

The denominator results from the evaluation of the integration constant $C$ whereas it is illustrated in [32] that the use of the inflection points $\pm P_{I}$, to simplify evaluation of the integrals, can be justified though either asymptotic analysis or energy arguments.

Debye arguments yield the differential equations

$$
\begin{aligned}
& \dot{x}_{+}=-p_{+-} x_{+}+p_{-+} x_{-} \\
& \dot{x}_{-}=-p_{-+} x_{-}+p_{+-} x_{+}
\end{aligned}
$$

quantifying the evolution of the respective dipole fractions. For implementation, the relations (10) can be simplified to the single differential equation

$$
\dot{x}_{+}=-p_{+-} x_{+}+p_{-+}\left(1-x_{+}\right)
$$

through the identity $x_{+}+x_{-}=1$.

The likelihoods of switching from positive to negative orientations, or vice versa, are respectively quantified by

$$
p_{+-}=\frac{1}{\mathcal{T}(T)} \frac{\int_{P_{I}}^{P_{I}+\epsilon} e^{-G\left(E, P_{I}, T\right) V / k T} d P}{\int_{P_{I}}^{\infty} e^{-G(E, P, T) V / k T} d P} \quad, \quad p_{-+}=\frac{1}{\mathcal{T}(T)} \frac{\int_{-P_{I}-\epsilon}^{-P_{I}} e^{-G\left(E,-P_{I}, T\right) V / k T} d P}{\int_{-\infty}^{-P_{I}} e^{-G(E, P, T) V / k T} d P}
$$

where $\epsilon$ is taken to be a small positive constant. The relaxation term $\mathcal{T}$ quantifies the frequency at which jumps are attempted whereas the remainder of the definition characterizes the probability of achieving the energy required to exit respective potential wells. It is detailed in [32] that this probability increases when the relative thermal energy $k T / V$ approaches the Gibbs energy $G$. For regimes in which $k T / V<<G$, it is also illustrated that the average local polarization relation (8) asymptotically limits to the expressions (3) or (4) employed for negligible thermal relaxation. 
The polarization relations (4) and (8) are derived at the lattice level and hence are mesoscopic in nature. For single crystal, homogeneous materials with uniform effective fields, the relations can be extrapolated throughout the material to yield constitutive models quantifying the hysteretic and nonlinear dependence of the bulk polarization $P$ on input fields $E$. Macroscopic models constructed in this manner retain the sharp transition through the coercive point exhibited by the kernels (4) or (8). While this models the physical behavior of certain single crystal compounds such as $\mathrm{BaTiO}_{3}-$ e.g., see page 76 of [21] — the transition is steeper than that typically exhibited by nonhomogeneous, polycrystalline compounds. To provide macroscopic $E-P$ models appropriate for this broader class of compounds, stochastic homogenization techniques utilizing the mesoscopic relations (4) or (8) as kernels are considered in the next section.

\subsection{Macroscopic Polarization Models}

The local average polarization relations (4) and (8) were derived under the assumption of a uniform lattice and constant effective field $E_{e}=E$. Hence they do not incorporate variability in the lattice due to nonuniform grain structure, material nonhomogeneities and defects, and variable stress fields nor do they include the effects of nonuniform interaction or effective fields. These effects can in theory be incorporated through micromechanical models utilizing extended energy relations $[5,6,16]$. However, this produces models whose complexity precludes bulk material characterization, transducer design or model-based control design. Alternatively, one can assume that certain parameters in the mesoscopic energy relations are manifestations of underlying stochastic distributions rather than constant values as posited for single crystals having uniform lattices. Stochastic homogenization in this manner produces macroscopic models which retain energy characteristics but are sufficiently low-order to permit implementation.

As noted in [32], variability in the lattice can be incorporated by considering the parameters $P_{R}, P_{I}$ or

$$
E_{c}=\eta\left(P_{R}-P_{I}\right)
$$

to be distributed, whereas variable interaction fields can be incorporated by considering effective fields $E_{e}$ to be distributed about the applied field $E$. In this section, we summarize the models of [32] based on a priori choices for the distributions whereas in Section 3, we consider the development of models having general densities estimated through fits to experimental data.

As illustrated in Figure 2, the coercive field $E_{c}$ is nonnegative which constitutes a constraint on the underlying density. Two a priori choices which enforce this constraint are normal distributions truncated to include only nonnegative values and lognormal distributions. The latter are employed in [32] which yield the density

$$
\nu_{1}\left(E_{c}\right)=c_{1} e^{-\left[\ln \left(E_{c} / \bar{E}_{c}\right) / 2 c\right]^{2}}
$$

where $c_{1}, c$ and $\bar{E}_{c}$ are positive constants. It is illustrated in [9] that if $c$ is small compared with $\bar{E}_{c}$, the mean and variance for the distribution have the approximate values

$$
\left\langle E_{c}\right\rangle \approx \bar{E}_{c} \quad, \quad \sigma \approx 2 \bar{E}_{c} c .
$$

It is illustrated in the examples of Section 4 that (14) provides initial estimates for the parameters $\bar{E}_{c}$ and $c$ based on measured properties of the data. We note that lognormal distributions of the form (13) are employed when quantifying the distribution of the critical field parameter $H_{k}$ in Preisach models used to model hard magnetic materials [9] which provides additional motivation for their consideration in the present setting.

Secondly, we consider variations in the effective field at the lattice level. As noted in [1, 20,30,31], the applied field $E$ is augmented by an interaction field $E_{i}$ due to neighboring dipoles as well as 
certain electromechanical interactions. While microelectric energy analysis can quantify some of these mechanisms, the required complexity of subsequent models precludes transducer design or real time control implementation. Alternatively, we make the a priori assumption that effective fields $E_{e}=E+E_{i}$ are distributed about the applied field with an underlying density characteristic of the ferroelectric material under consideration. The models employed in [32] are based on the assumption that $E_{e}$ is normally distributed about $E$ with the density

$$
\nu_{2}\left(E_{e} ; E\right)=c_{2} e^{-\left(E-E_{e}\right)^{2} / 2 b^{2}} .
$$

The magnitude of the variance $b^{2}$ dictates the degree to which switching occurs prior to the remanence polarization. Large values of $b^{2}$ produce models with significant switching as the applied field $E$ is reduced to zero with correspondingly large changes in the slope of the hysteresis curve whereas small values of $b^{2}$ are employed when modeling the hysteretic response of materials exhibiting nearly linear $E-P$ behavior at remanence.

The resulting polarization model is

$$
[P(E)](t)=C \int_{0}^{\infty} \int_{-\infty}^{\infty}\left[\bar{P}\left(E+E_{i}, E_{c}, \xi\right)\right](t) e^{-E_{i}^{2} / 2 b^{2}} e^{-\left[\ln \left(E_{c} / \bar{E}_{c}\right) / 2 c\right]^{2}} d E_{i} d E_{c}
$$

where the kernel $\bar{P}$ is specified by (3), (4) or (8) and $\xi$ denotes the initial distribution of dipoles.

\subsection{Discrete Macroscopic Polarization Model}

To implement the model (16), it is necessary to approximate the integrals. As detailed in [32], this can be accomplished either by employing Gaussian quadrature routines constructed for infinite or semi-infinite domains or by exploiting the decay exhibited by the kernels to truncate the domains and employ Gauss-Legendre quadrature rules. Both strategies yield discrete models of the form

$$
[P(E)](t)=C \sum_{i=1}^{N_{i}} \sum_{j=1}^{N_{j}}\left[\bar{P}\left(E+E_{i_{j}} ; E_{c_{i}}, \xi_{i}\right)\right](t) e^{-E_{i_{j}}^{2} / b} e^{-\left[\ln \left(E_{c_{i}} / \bar{E}_{c}\right) / 2 c\right]^{2}} v_{i} w_{j}
$$

where $E_{i_{j}}, E_{c_{i}}$ denote the abscissas associated with respective quadrature formulae and $v_{i}, w_{j}$ are the respective weights - e.g., see pages 698-699 of [37].

For the characterization examples presented in Section 4, as well as in [32], we employ a composite, 4 point Gauss-Legendre quadrature rule constructed for truncated domains, and we illustrate that approach here. Consider first the approximation of the effective field integral. For a given threshold $\epsilon$ and index $N_{q}$, we let $[-L, L]$ denote the interval where $\left|e^{-E_{i}^{2}}\right| \geq \epsilon$ and consider the partition $h_{q}=-L+q h, h=2 L / N_{q}$. On each subinterval $\left[h_{q-1}, h_{q}\right]$, the quadrature points and weights are specified to be

$$
\begin{array}{lll}
E_{i_{q 1}}=h_{q-1}+h\left[\frac{1}{2}-\frac{\sqrt{15+2 \sqrt{30}}}{2 \sqrt{35}}\right] & w_{q 1}=\frac{49 h}{12(18+\sqrt{30})} \\
E_{i_{q 2}}=h_{q-1}+h\left[\frac{1}{2}-\frac{\sqrt{15-2 \sqrt{30}}}{2 \sqrt{35}}\right] & , & w_{q 2}=\frac{49 h}{12(18-\sqrt{30})} \\
E_{i_{q 3}}=h_{q-1}+h\left[\frac{1}{2}+\frac{\sqrt{15-2 \sqrt{30}}}{2 \sqrt{35}}\right] & w_{q 3}=\frac{49 h}{12(18-\sqrt{30})} \\
E_{i_{q 4}}=h_{q-1}+h\left[\frac{1}{2}+\frac{\sqrt{15+2 \sqrt{30}}}{2 \sqrt{35}}\right] & , & w_{q 4}=\frac{49 h}{12(18+\sqrt{30})} .
\end{array}
$$


Similar analysis yields the quadrature points $E_{c_{p k}}$ and weights $v_{p k}$ for the approximation of the coercive field integral on a grid with $N_{p}$ subintervals. In this case, $N_{i}=4 N_{p}, N_{j}=4 N_{q}$, and the formulation (17) has the specific form

$$
[P(E)](t)=C \sum_{p=1}^{N_{p}} \sum_{k=1}^{4}\left(\sum_{q=1}^{N_{q}} \sum_{\ell=1}^{4}\left[\bar{P}\left(E_{i_{q \ell}}+E ; E_{c_{p k}}, \xi_{q \ell}\right)\right](t) e^{-E_{i_{q \ell}}^{2} / 2 b^{2}} w_{q \ell}\right) e^{-\left[\ln \left(E_{c_{p k}} / \bar{E}_{c}\right) / 2 c\right]^{2}} v_{p k} .
$$

Details regarding the discrete formulation model and a highly efficient implementation algorithm are provided in [32].

We note that for the linear kernel $\bar{P}$ given by (3) or (4), construction of the model using this a priori choice of normal and lognormal distributions entails the identification of 6 parameters. The parameters $P_{R}$ and $\eta$ arise in the definition of the kernel whereas $\bar{E}_{c}, c, b$ and $C$ are associated with the construction of the density functions $\nu_{1}$ and $\nu_{2}$. We note that for this choice of kernel, $P_{R}$ and $C$ both scale final polarization values and hence can be combined into a single effective parameter. Thus for materials in which the normal and lognormal densities provide sufficient accuracy, the low number and physical nature of parameters makes the model highly efficient to construct and update.

\section{Polarization Model with General Densities}

Whereas certain physical arguments motivate consideration of the normal and lognormal distributions for the effective and coercive fields, we rely primarily on their mathematical attributes including the easily quantified relations between properties of the data - including the coercivity and degree of pre-remanence switching - and mean and variance properties of the densities. Moreover, as long as the densities enforce positive arguments for the coercive field and exhibit certain decay properties, there is no physical reason why normal and lognormal functions are inherently preferable to general densities which provide substantially greater flexibility for model construction. We thus consider the formulation of the polarization model in terms of general densities and indicate techniques suitable for estimating the densities. The attributes of models constructed using general densities are compared with those based on the a priori assumption of normal and lognormal distributions in Section 4 .

\subsection{Polarization Model}

We consider general densities $\nu_{1}$ and $\nu_{2}$ which satisfy the conditions

(i) $\quad \nu_{1}(x)$ defined for $x>0$,

(ii) $\nu_{2}(-x)=\nu_{2}(x)$,

(iii) $\left|\nu_{1}(x)\right| \leq c_{1} e^{-a_{1} x} \quad, \quad\left|\nu_{2}(x)\right| \leq c_{2} e^{-a_{2} x}$

for nonnegative $c_{1}, a_{1}, c_{2}, a_{2}$. The restricted domain in (i) reflects the fact that the coercive field $E_{c}$ is positive whereas the symmetry enforced in the effective field through (ii) yields the symmetry observed in low-field Rayleigh loops. Hypothesis (iii) incorporates the physical observation that the coercive and interactions fields decay as a function of distance and guarantees that integration against the piecewise linear kernel yields finite polarization values.

The general polarization model can then be formulated as

$$
\begin{aligned}
{[P(E)](t) } & =\int_{0}^{\infty} \int_{-\infty}^{\infty} \nu_{1}\left(E_{c}\right) \nu_{2}\left(E_{i}\right)\left[\bar{P}\left(E+E_{i} ; E_{c}, \xi\right)\right](t) d E_{i} d E_{c} \\
& =\int_{0}^{\infty} \int_{-\infty}^{\infty} \nu\left(E_{c}, E_{i}\right)\left[\bar{P}\left(E+E_{i} ; E_{c}, \xi\right)\right](t) d E_{i} d E_{c}
\end{aligned}
$$


where $\bar{P}$ is specified in (3), (4) or (8). Whereas formulation of the model in terms of the twodimensional density $\nu$ is more general, retention of the components $\nu_{1}$ and $\nu_{2}$ can facilitate subsequent implementation.

The approximation of the integrals yields the discrete model

$$
[P(E)](t)=\sum_{i=1}^{N_{i}} \sum_{j=1}^{N_{j}} \nu_{1}\left(E_{c_{i}}\right) \nu_{2}\left(E_{i_{j}}\right)\left[\bar{P}\left(E+E_{i_{j}} ; E_{c_{i}}, \xi\right)\right](t) v_{i} w_{j}
$$

where $v_{i}, w_{j}$ denote the quadrature points and $E_{c_{i}}, E_{i_{j}}$ are the abscissas for the given quadrature rule.

To illustrate attributes of the discrete model (22), we compare it with the model (17) derived under the a priori assumption that

$$
\begin{aligned}
& \nu_{1}\left(E_{c}\right)=c_{1} e^{-\left[\ln \left(E_{c} / \bar{E}_{c}\right) / 2 c\right]^{2}} \\
& \nu_{2}=c_{2} e^{-E_{i}^{2} / b}
\end{aligned}
$$

The construction of (17) requires the identification of the 5 parameters $\eta, C, \bar{E}_{c}, c$ and $b$ once $P_{R}$ is combined with $C$. The construction of the model (22) in terms of $\nu_{1}$ and $\nu_{2}$ requires the identification of the $N_{i}+N_{j}+1$ parameters $\left[\nu_{1}\left(E_{c_{1}}\right), \cdots, \nu_{1}\left(E_{c_{N_{i}}}\right)\right],\left[\nu_{2}\left(E_{i_{1}}\right), \cdots, \nu_{2}\left(E_{i_{N_{j}}}\right)\right]$ and $\eta$. If one employs the product density $\nu$, discretization yields $N_{i} \cdot N_{j}+1$ parameters since one must identify all of the values $\nu\left(E_{c_{i}}, E_{i_{j}}\right)$ in this formulation. Since one typically requires $N_{i}$ and $N_{j}$ on the order of 20 to 80 to achieve convergence, the identification of the general densities using either the isolated densities $\nu_{1}$ and $\nu_{2}$, or the product density $\nu$, requires highly efficient optimization and parameter estimation techniques.

The advantage gained through this effort is the construction of models which are highly accurate for a wide range of drive regimes. It is important to note that whereas the general density model (23) may be more expensive to construct than the model (17), the implementation of the two models requires identical overhead since both simply employ multiplication of $N_{i} \times 1$ and $N_{j} \times 1$ vectors. Hence they will be equally efficient for model-based control design.

\subsection{Estimation of $\nu_{1}$ and $\nu_{2}$ through Constrained Optimization}

To estimate the $N_{i}+N_{j}+1$ parameters $\left[\nu_{1}\left(E_{c_{1}}\right), \cdots, \nu_{1}\left(E_{c_{N_{i}}}\right)\right],\left[\nu_{2}\left(E_{i_{1}}\right), \cdots, \nu_{2}\left(E_{i_{N_{j}}}\right)\right]$ and $\eta$, we consider a least squares fit to data $\left(\widehat{E}_{k}, \widehat{P}_{k}\right), k=1, \cdots, N_{d}$. The accuracy of resulting models will be improved if the data is chosen to include all drive regimes under consideration and, in general, inclusion of a highly varied set of drive regimes will provide more comprehensive characterization of the densities. For example, identification of the densities solely based on symmetric major loop data will provide a model which has moderate accuracy when predicting biased minor loops whereas identification using data that includes biased drive level data will provide a model with improved accuracy in these regimes.

To formulate the constrained optimization problem, we define $\theta=\left[\eta, \nu_{1}\left(E_{c_{1}}\right), \cdots, \nu_{1}\left(E_{c_{N_{i}}}\right)\right.$, $\left.\nu_{2}\left(E_{i_{1}}\right), \cdots, \nu_{2}\left(E_{i_{N_{j}}}\right)\right] \in \mathbb{R}^{N_{i}+N_{j}+1}$ and let $P\left(\widehat{E}_{k} ; \theta\right)$ denote the modeled parameter-dependent polarization values given by (22) for the $N_{d}$ input field values $\widehat{E}_{k}$. The constrained optimization problem can then be formulated as follows: find $\theta \in \mathbb{R}^{N_{i}+N_{j}+1}$ which minimizes

$$
f(\theta)=\frac{1}{2}\left\|P\left(\widehat{E}_{k} ; \theta\right)-\widehat{P}_{k}\right\|^{2}
$$


subject to

$$
\theta_{i} \geq 0 \text {. }
$$

Here $\|\cdot\|$ denotes the Euclidean norm in $\mathbb{R}^{N_{d}}$. For the general formulation (24), optimization can be performed using the Matlab routine fmincom.m, or other optimization routines which enforce the positivity constraint for moderate values of $N_{i}$ and $N_{j}$ - e.g., $N_{i}=N_{j}=80$. We note that solution of (24) is analogous to the technique employed in [34] for estimating parameters in discretized Preisach models.

\subsection{Estimation of $\nu$ - Direct Solution Algorithm}

Alternatively, one can consider the more general formulation

$$
[P(E)](t)=\int_{0}^{\infty} \int_{-\infty}^{\infty} \nu\left(E_{c}, E_{i}\right)\left[\bar{P}\left(E+E_{i} ; E_{c}, \xi\right)\right](t) d E_{i} d E_{c}
$$

where $\nu: \mathbb{R}^{2} \rightarrow \mathbb{R}$ is a general density function to be estimated for a given compound, and discretized relation

$$
[P(E)](t)=\sum_{i=1}^{N_{i}} \sum_{j=1}^{N_{j}} \nu\left(E_{c_{i}}, E_{i_{j}}\right)\left[\bar{P}\left(E+E_{i_{j}} ; E_{c_{i}}, \xi\right)\right](t) v_{i} w_{j} .
$$

This formulation has the advantage that it yields a linear system in terms of $\nu$. Furthermore, it yields a quadratic programming problem when the discrete model (26) is employed in a least squares setting but it comes at the cost of estimating $N_{i} \cdot N_{j}$ unknowns as compared with the $N_{i}+N_{j}+1$ variables required for the nonlinear constrained optimization problem discussed in Section 3.2 - we assume here that $\eta$ can be estimated directly from the slope $\frac{d P}{d E}$ of data after switching.

In the absence of thermal relaxation, evaluation of (3) at the measured input field values $\widehat{E}_{k}$ yields

$$
P\left(E_{k}\right)=\sum_{j=1}^{N_{j}} \sum_{i=1}^{N_{i}}\left[\frac{\widehat{E}_{k}+E_{i_{j}}}{\eta}+P_{R} \delta\left(\widehat{E}_{k} ; E_{c_{i}}, E_{i_{j}}\right)\right] \nu\left(E_{c_{i}}, E_{i_{j}}\right) v_{i} w_{j}
$$

for $k=1, \cdots, N_{d}$. For clarity, we note the dependence of $\delta$ on the coercive field quadrature points.

To formulate (27) as a linear system, we define the $N_{i} \times N_{j}$ matrices $A_{k}$ and $\Phi$ to have components

$$
\begin{aligned}
& {\left[A_{k}\right]_{i j}=\left[\frac{\widehat{E}_{k}+E_{i_{j}}}{\eta}+P_{R} \delta\left(\widehat{E}_{k} ; E_{c_{i}}, E_{i_{j}}\right)\right] v_{i} w_{j}} \\
& {[\Phi]_{i j}=\nu\left(E_{c_{i}}, E_{i_{j}}\right) .}
\end{aligned}
$$

For $N=N_{i} \cdot N_{j}$, we define the $N \times 1$ vector $\theta$ and $1 \times N$ vector $a_{k}$ by

$$
\theta=\operatorname{vec}(\Phi) \quad, \quad a_{k}=\left[\operatorname{vec}\left(A_{k}\right)\right]^{T}
$$

where 'vec' denotes the vector concatenation of the respective matrices. Additionally, the $N_{d} \times 1$ vectors $\mathcal{P}$ and $\widehat{\mathcal{P}}$ are defined componentwise by

$$
[\mathcal{P}]_{k}=P\left(\widehat{E}_{k}\right) \quad, \quad[\widehat{\mathcal{P}}]_{k}=\widehat{P}_{k} .
$$

Finally, the $N_{d} \times N$ matrix $A$ is defined row-wise by

$$
[A]_{k}=a_{k} .
$$


The polarization model (27) can then be formulated as the linear system

$$
A \theta=\mathcal{P}
$$

and the least squares problem used to identify the density values $\theta$ given measurements $\left\{\widehat{E}_{k}, \widehat{P}_{k}\right\}, k=$ $1, \cdots, N_{d}$, is the following: minimize

$$
f(\theta)=\frac{1}{2}\|A \theta-\widehat{\mathcal{P}}\|^{2}
$$

subject to

$$
\theta_{j} \geq 0, j=1, \cdots, N
$$

Because the minimum is unaffected by a constant shift, this is equivalent to the problem

$$
\begin{aligned}
& \min _{\theta} f(\theta), f(\theta)=\frac{1}{2} \theta^{T} A^{T} A \theta-\widehat{\mathcal{P}}^{T} A \theta \\
& \text { subject to } \theta_{j} \geq 0, j=1, \cdots, N .
\end{aligned}
$$

To solve (33), we employ the same technique used in [28] for identifying density functions in Preisach models. Consider the singular value decomposition

$$
A^{T} A=U S V^{T}
$$

where $U=V$ since $A^{T} A$ is symmetric, and the $N \times N$ diagonal matrix $S$ is comprised of the singular values of $A^{T} A$. Furthermore, it is noted that the column vectors $u_{i}$ of $U$ satisfy the orthogonality condition

$$
u_{i}^{T} u_{j}=\delta_{i j}
$$

where $\delta_{i j}$ denotes the Kronecker delta.

We consider the case $\operatorname{rank}(A)=\operatorname{rank}(S)=q<\min \left\{N, N_{d}\right\}$ which arises when considering fine discretizations to fully resolve fine-scale properties of the hysteretic response. For this case, we eliminate the rows and columns of $S$ and $U$ corresponding to zero singular values to obtain the $q \times q$ matrix $\widehat{S}$ and $N \times q$ matrix $\widehat{U}$. If instability due to ill-conditioning induced by small singular values is of concern, one can alternatively retain singular values greater than a specified threshold $\varepsilon$ along with corresponding components in $U$ - this is the basis for the truncated SVD (TSVD) techniques.

Because

$$
A^{T} A=\widehat{U} \widehat{S} \widehat{U}^{T} \quad, \quad \widehat{U}^{T} \widehat{U}=I,
$$

the minimization problem (33) can be reformulated as

$$
\begin{aligned}
& \min _{x} f(x), f(x)=\frac{1}{2} x^{T} \widehat{S} x-\widehat{\mathcal{P}}^{T} A \widehat{U} x \\
& \text { subject to } g(x)=\widehat{U} x \geq 0
\end{aligned}
$$

where $x=\widehat{U}^{T} \theta$ and hence $\theta=\widehat{U} x$. The quadratic programming problem (36) can be solved using the Matlab routine quadprog.m to obtain $x^{*}$, and a corresponding solution $\theta^{*}$ to (33) is then given by $\theta^{*}=\widehat{U} x^{*}$. 


\subsection{Estimation of $\nu$ - Regularized Solution}

It is shown in [29] that the polarization model (25) is an integral equation having a compact integral operator. Hence the inverse problem of determining $\nu$ given data measurements is ill-posed both with regard to the existence of a unique solution and the continuous dependence of $\nu$ on the data. It is illustrated in the validation example of Section 4.3 that this ill-posedness is manifested in the discretized formulation (26) through a loss in accuracy as discretization limits are increased. This motivates the consideration of regularized least squares formulations for determining $\nu$.

As detailed in [29], Tikhonov regularization is imposed by replacing the least squares functional (32) by the augmented functional

$$
f_{\alpha}(\theta)=\frac{1}{2}\|A \theta-\widehat{\mathcal{P}}\|^{2}+\frac{\alpha}{2}\|\theta\|^{2}
$$

which shifts the spectrum of $A^{T} A$ to $A^{T} A+\alpha I$ to avoid the deleterious effects of small singular values. The constrained minimization problem in this case is

$$
\begin{aligned}
& \min _{\theta} f_{\alpha}(\theta), f(\theta)=\frac{1}{2}\|A \theta-\widehat{\mathcal{P}}\|^{2}+\frac{\alpha}{2}\|\theta\|^{2} \\
& \text { subject to } \theta_{j} \geq 0, j=1, \ldots, N .
\end{aligned}
$$

Techniques for choosing $\alpha$ to avoid oversmoothing solutions as well as a solution algorithm for (38) can be found in Vogel [36].

\section{Model Validation}

To illustrate attributes of the model and the flexibility provided by the formulation in terms of general densities, we consider the characterization of PZT5H and PZT4. The PZT5H example illustrates the capability of the model to accurately characterize biased minor loops under quasistatic drive conditions. An important attribute of the model is its capability for ensuring closure of the loops under such conditions in accordance with experimental behavior of the materials. The PZT4 example illustrates the predictive capabilities for the model for a hard PZT compound. In both cases, we employ the polarization kernel (3) or (4) since relaxation mechanisms are negligible. For operating regimes in which relaxation must be accommodated, the kernel (8) would be employed in an analogous manner.

\subsection{PZT4 Characterization}

We consider first the characterization of constitutive nonlinear and hysteresis exhibited by PZT4. Data was collected from a $3.81 \mathrm{~cm} \times 0.635 \mathrm{~cm} \times 0.381 \mathrm{~cm}$ wafer at $200 \mathrm{mHz}$ to minimize frequency effects. The measured $E-P$ relations corresponding to peak input voltages ranging from $600 \mathrm{~V}$ to $1800 \mathrm{~V}$ are plotted in Figure 3. Note that the relation between $E$ and $V$ can be approximated by $E=V / h$ where $h=3.81 \times 10^{-4}$ is the thickness of the wafer. To demonstrate properties of the model using both the normal/lognormal and general densities and differing identification strategies, we consider four regimes:

(i) normal/lognormal densities estimated using all four data sets;

(ii) general densities estimated using all four data sets;

(iii) normal/lognormal densities estimated using the $1800 \mathrm{~V}$ data set;

(iv) general densities estimated using the $1800 \mathrm{~V}$ data set.

In all cases, convergence was achieved with $N_{p}=N_{q}=20$ which yields the parameter limits $N_{i}=$ $N_{j}=80$. 


\section{Lognormal/Normal Densities — Identification Using 600 V - 1800 V Data}

To provide a baseline for comparison, we take $\nu_{1}$ and $\nu_{2}$ as the lognormal and normal functions specified in (23) and employ four data sets - $600 \mathrm{~V}, 1000 \mathrm{~V}, 1200 \mathrm{~V}$ and $1800 \mathrm{~V}$ - when estimating the parameters $P_{R}, \eta, \bar{E}_{c}, c, b$ and $C$. The resulting model response, is compared with the data in Figure 3. The coercive and effective field densities $\nu_{1}$ and $\nu_{2}$ are plotted in Figure 4 . It is observed that the model is reasonably accurate throughout the drive range which is to be expected since all four data sets were employed when estimating parameters. The accuracy is quantified by the residual

$$
\mathcal{R}=\left[\frac{1}{N_{d}} \sum_{k=1}^{N_{d}}\left|P\left(\widehat{E}_{k}\right)-\widehat{P}_{k}\right|^{2}\right]^{1 / 2}
$$

between the modeled response and polarization data at the specified input field values which, as summarized in Table 1 , is 0.0226 .

\begin{tabular}{l|ccc} 
& (i) & (ii) & (iii) \\
\hline General Densities & 0.0176 & 0.0383 & 0.0113 \\
Lognormal/Normal Densities & 0.0226 & 0.0533 & 0.0164
\end{tabular}

Table 1: Residuals $\mathcal{R}$ given by (39). (i) Identification and residuals over four data sets (600 V - $1800 \mathrm{~V}$ data). (ii) Identification using $1800 \mathrm{~V}$ data - residual for all four data sets. (iii) Identification using $1800 \mathrm{~V}$ data — residual for $1800 \mathrm{~V}$ data.
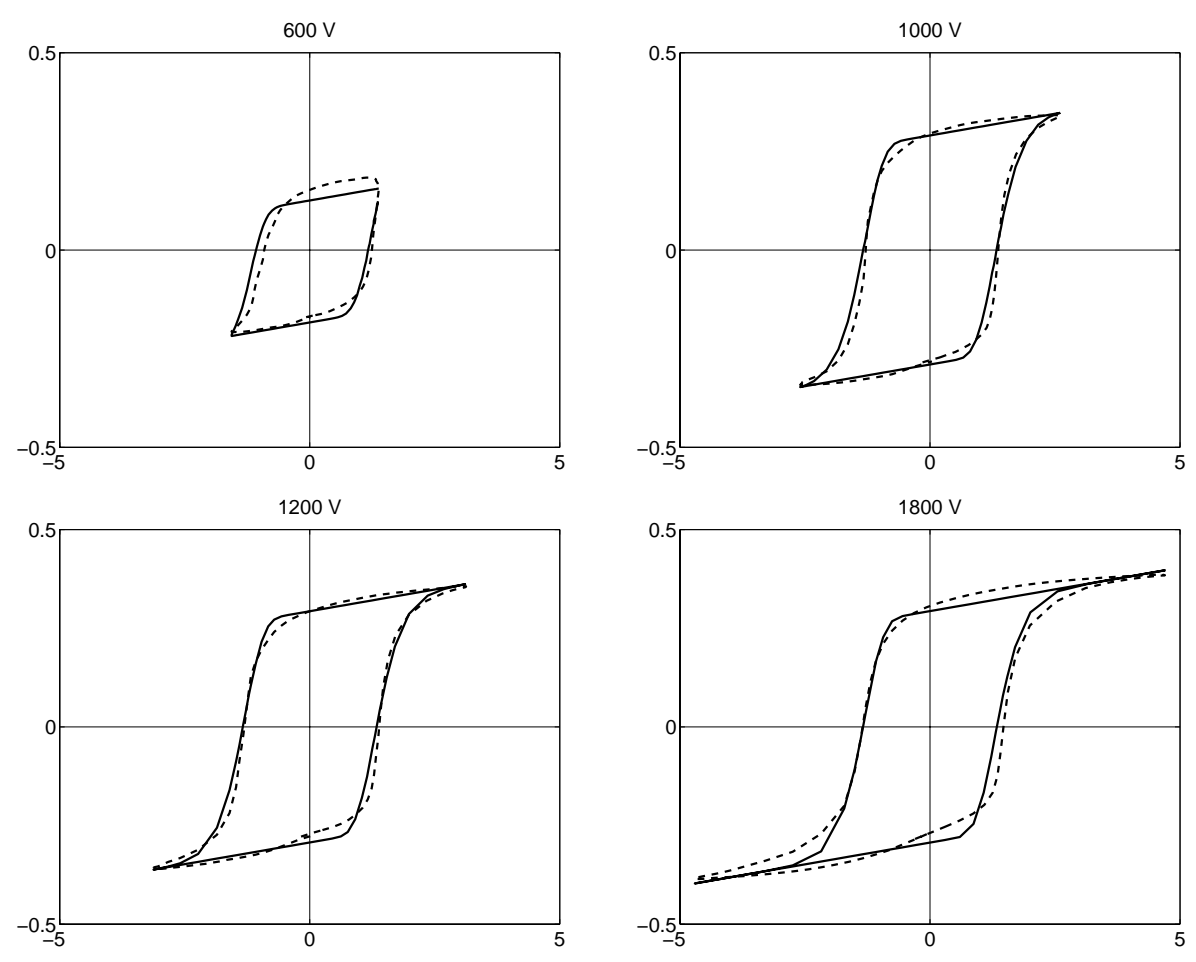

Figure 3: PZT4 data $(---)$ and model predictions (-) with lognormal/normal densities for $\nu_{1}$ and $\nu_{2}$ and parameters estimated through a least squares fit to all four data sets. Abscissas: electric field $(\mathrm{MV} / \mathrm{m})$, ordinates: polarization $\left(\mathrm{C} / \mathrm{m}^{2}\right)$. 

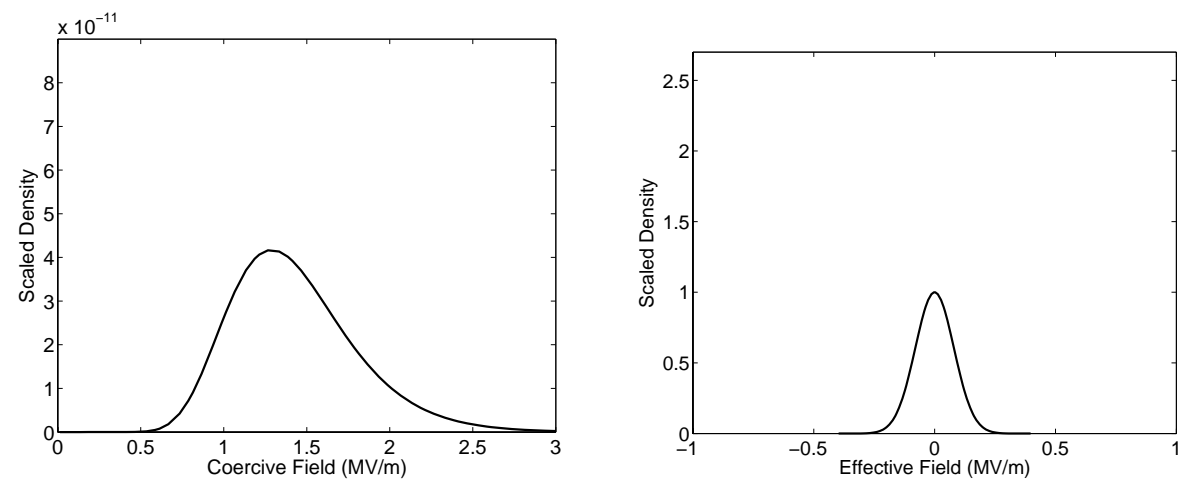

Figure 4: Lognormal coercive field density $\nu_{1}$ and effective field density $\nu_{2}$ given by (23) with parameters estimated through a least squares fit to $600 \mathrm{~V}-1800 \mathrm{~V}$ data.

\section{General Densities - Identification Using 600 V - 1800 V Data}

We now assume that $\nu_{1}$ and $\nu_{2}$ are general functions satisfying the hypotheses (20) and we estimate the $N_{i}+N_{j}+1$ parameters $\left[\nu_{1}\left(E_{c_{1}}\right), \cdots, \nu_{1}\left(E_{c_{N_{i}}}\right)\right],\left[\nu_{2}\left(E_{i_{1}}\right), \cdots, \nu_{2}\left(E_{i_{N_{j}}}\right)\right]$ and $\eta$, through the solution of the constrained least squares problem (24). The resulting model fits are plotted in Figure 5 and the estimated densities are plotted in Figure 6. A comparison between Figures 3 and 5 indicates that the model with general densities is more accurate as quantified by the residual of 0.0176 which is approximately $60 \%$ that obtained using the a priori choice of lognormal and normal densities
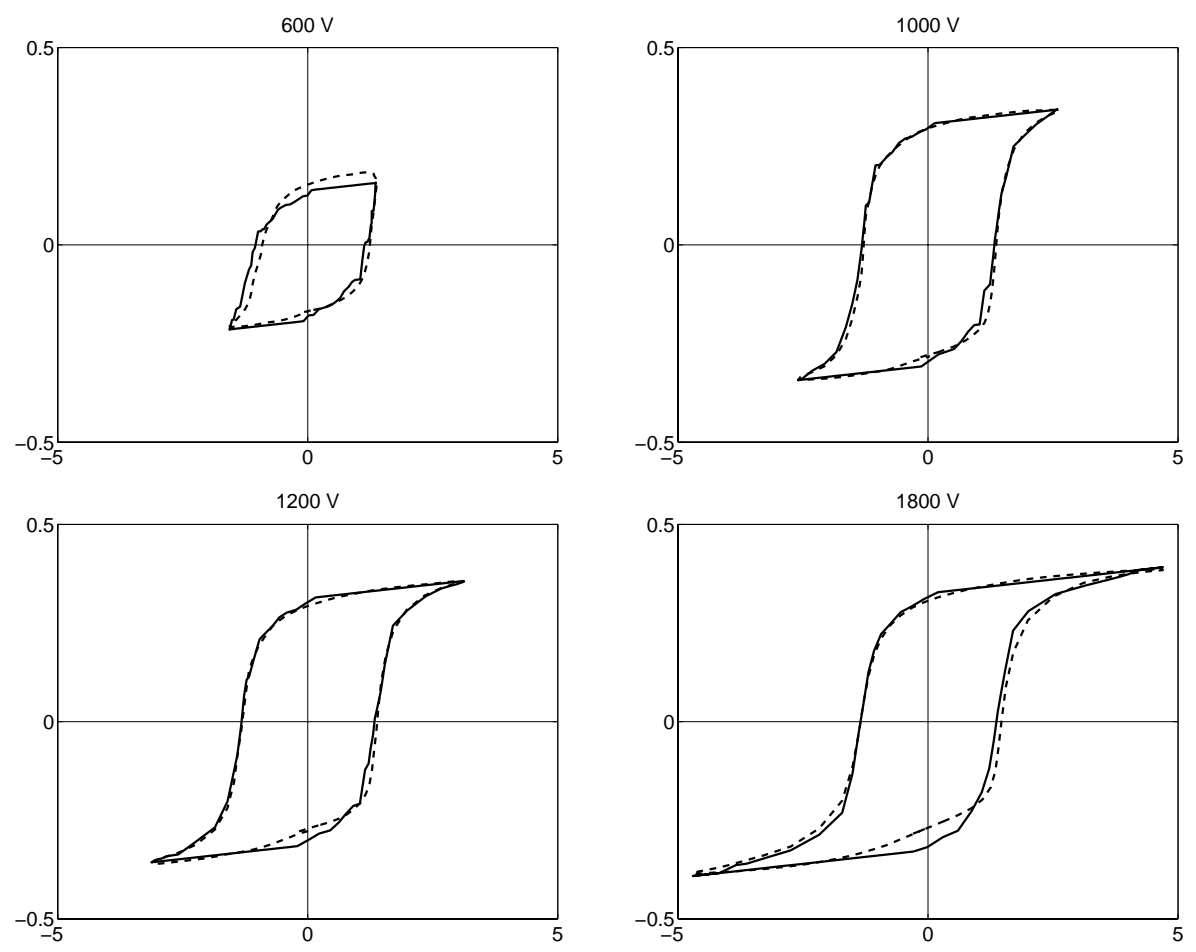

Figure 5: PZT4 data $\left(--_{-}\right)$and model predictions (- ${ }_{-}$obtained with the general densities $\nu_{1}$ and $\nu_{2}$ estimated through a constrained least squares fit to all four data sets. Abscissas: electric field $(\mathrm{MV} / \mathrm{m})$, ordinates: polarization $\left(\mathrm{C} / \mathrm{m}^{2}\right)$. 

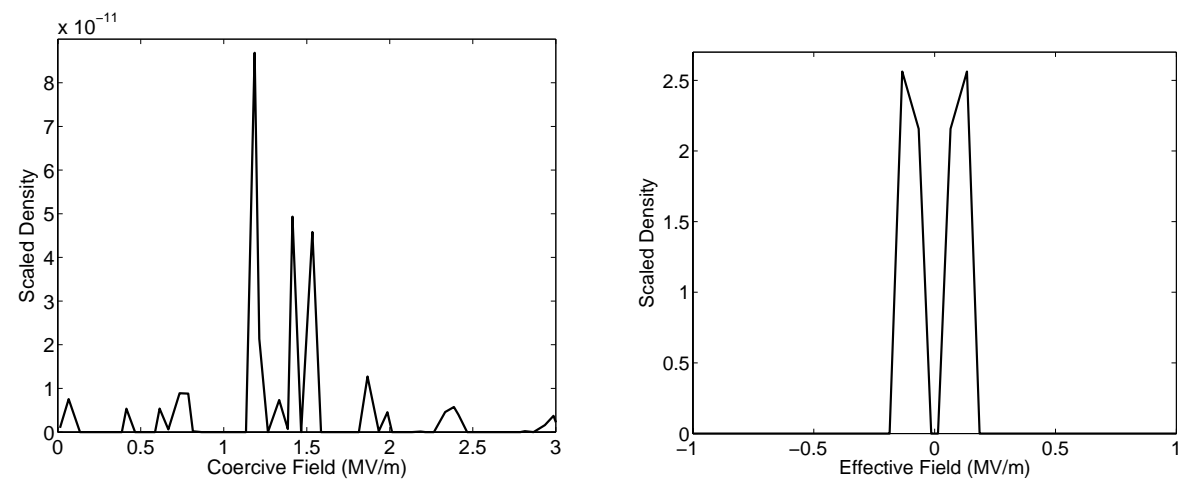

Figure 6: Coercive field density $\nu_{1}$ and effective field density $\nu_{2}$ estimated using all four data sets.

- see Table 1. A comparison between the coercive and effective field densities and those plotted in Figure 4 illustrates that the general densities have a qualitative shape similar to the lognormal and normal densities but are significantly less regular and do not exhibit the same monotonicity.

\section{Lognormal/Normal Densities — Identification Using 1800 V Data}

The third case consists of identification of the lognormal and normal densities using the $1800 \mathrm{~V}$ data and subsequent model predictions at the $600 \mathrm{~V}, 1000 \mathrm{~V}$ and $1200 \mathrm{~V}$ levels. This yielded the model responses plotted in Figure 7. A comparison between these results and those plotted in Figure 3 illustrates a slightly improved fit at $1800 \mathrm{~V}$ but a significantly worse fit at $600 \mathrm{~V}$. This is
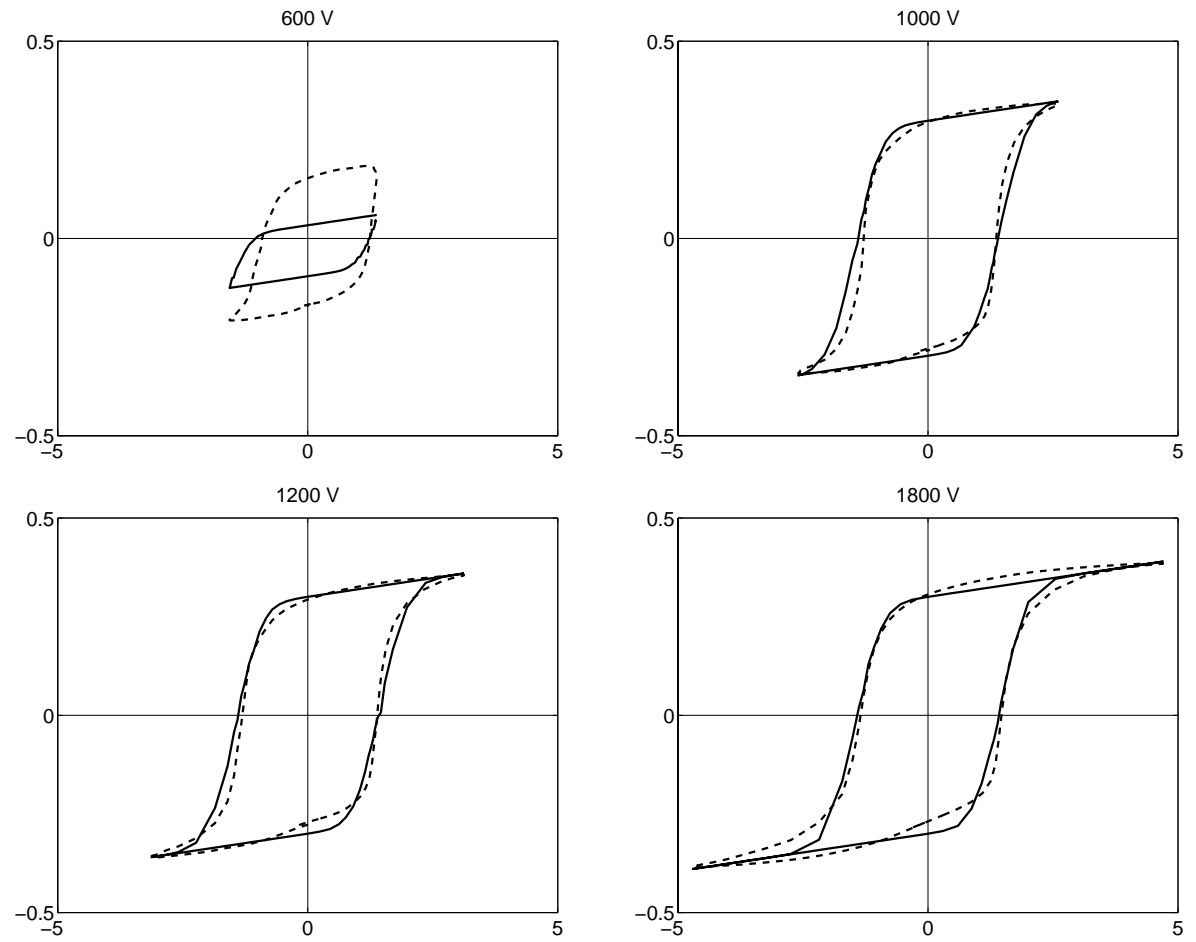

Figure 7: PZT4 data (-- ) and model predictions (-) with lognormal/normal densities for $\nu_{1}$ and $\nu_{2}$ and parameters estimated through a least squares fit to the $1800 \mathrm{~V}$ data. Abscissas: electric field $(\mathrm{MV} / \mathrm{m})$, ordinates: polarization $\left(\mathrm{C} / \mathrm{m}^{2}\right)$. 
reflected in the residual of 0.0533 which is more than a factor of two worse than when all four data sets are employed for parameter estimation. This illustrates that while the model has substantial predictive capabilities, improved performance can be obtained by estimating parameters using data from a range of drive regimes.

\section{General Densities - Identification Using 1800 V Data}

To complete the repertoire, we consider the identification of the general densities $\nu_{1}$ and $\nu_{2}$ through solution of the constrained minimization problem (24) using only the $1800 \mathrm{~V}$ data. A comparison of the resulting model fits in Figure 8 with those in Figure 5 illustrates an improved and highly accurate fit to the $1800 \mathrm{~V}$ data, reasonable predictions at $1000 \mathrm{~V}$ and $1200 \mathrm{~V}$, and only moderately accurate predictions at $600 \mathrm{~V}$. As noted in Table 1, the residual of 0.0383 over all four data sets is larger than those obtained with either general densities or the a priori choice of lognormal/normal densities when all four data sets are employed for identification but is significantly smaller than the lognormal/normal model fit when identifying parameters using only the $1800 \mathrm{~V}$ data. This same tendency is noted in the residuals over only the $1800 \mathrm{~V}$ data.
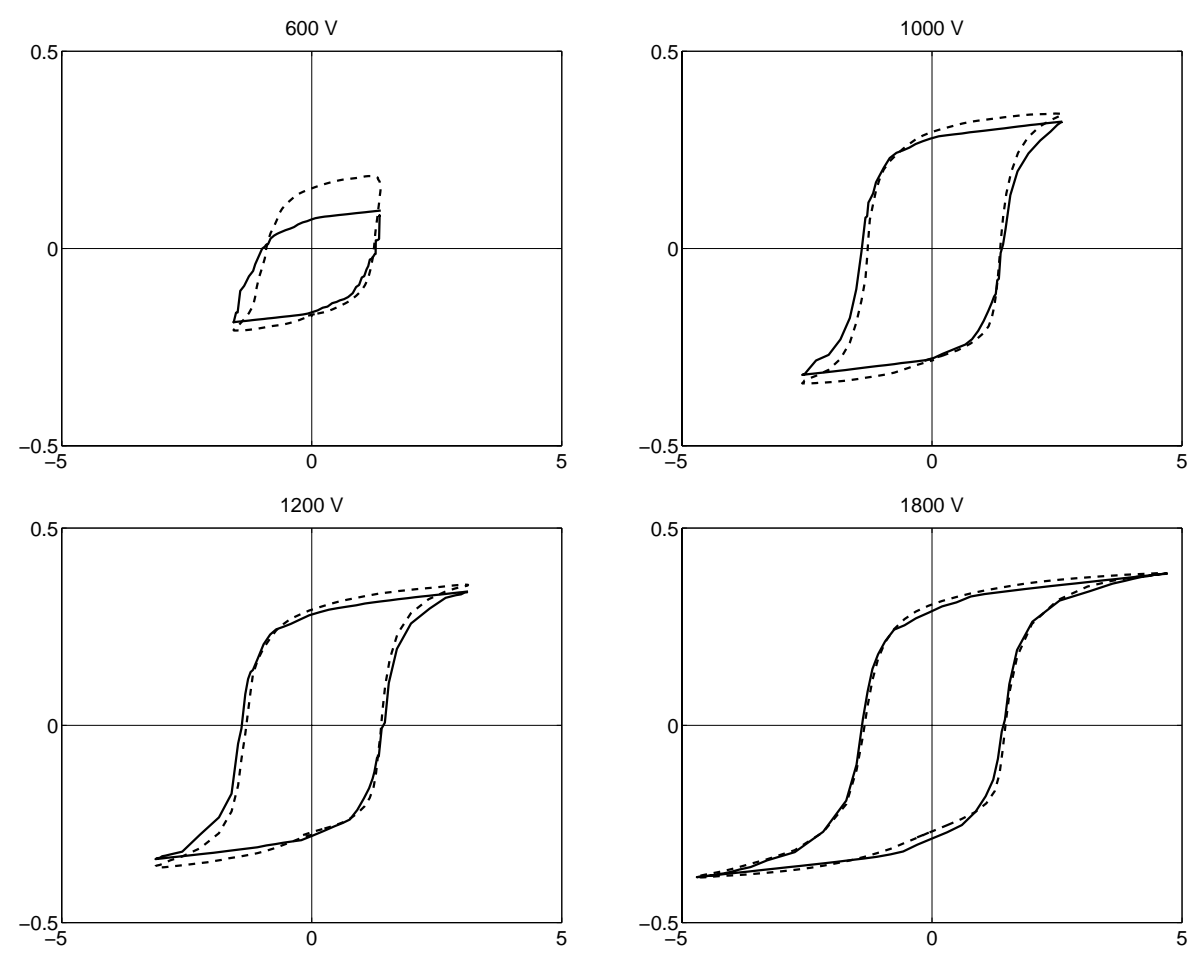

Figure 8: PZT4 data (-- ) and model predictions (- ${ }_{-}$) obtained with the general densities $\nu_{1}$ and $\nu_{2}$ estimated through a constrained least squares fit to the $1800 \mathrm{~V}$ data. Abscissas: electric field $(\mathrm{MV} / \mathrm{m})$, ordinates: polarization $\left(\mathrm{C} / \mathrm{m}^{2}\right)$.

\subsection{PZT5H Characterization - Individual Densities $\nu_{1}$ and $\nu_{2}$}

The second compound we consider is PZT5H which exhibits the hysteretic and nonlinear behavior shown in Figure 1. In this case, we illustrate the capability of the model to accurately quantify biased minor loop behavior in quasistatic drive regimes - data was collected at $0.2 \mathrm{~Hz}$. We again consider four cases to illustrate the performance of the model constructed with general densities as compared with the model employing lognormal/normal densities: 
(i) General densities — identified through fit to all 7 loops;

(ii) Normal/lognormal densities — identified through fit to all 7 loops;

(iii) General densities - identified through fit to symmetric major loop and Rayleigh loop;

(iv) Normal/lognormal densities — identified through fit to major loop and Rayleigh loop. Convergence was achieved in all cases with a total of $N_{i}=N_{j}=80$ quadrature points.

\section{General Densities - Identification Using Full Data Set}

We first consider the model performance when the full set of data (all 7 loops) was used to estimate the $N_{i}+N_{j}+1$ parameters $\left[\eta, \nu_{1}\left(E_{c_{1}}\right), \cdots, \nu_{1}\left(E_{c_{N_{i}}}\right), \nu_{2}\left(E_{i_{1}}\right), \cdots, \nu_{2}\left(E_{i_{N_{j}}}\right)\right]$ through solution of the nonlinear constrained optimization problem (24) which enforces positivity in all $N_{i}+N_{j}+1$ parameters and symmetry in $\nu_{2}$. The resulting model fit is plotted in Figure 9 and the densities $\nu_{1}$ and $\nu_{2}$ are shown in Figure 10. It is observed that the model accurately characterizes both the symmetric major loop behavior and the biased minor loop responses including the nested biased minor loop. The slight oscillations in the model fit preceding saturation result from the optimization of the densities to accommodate minor loop behavior and are not a manifestation of numerical stability for large stepsizes in the input fields. For later comparison with fits obtained using the lognormal/normal densities, the residual (39) is noted to be $\mathcal{R}=0.0057$ as summarized in Table 2 .

It should be noted that when traversing from the endpoint of the biased minor loop to the saturation value, no a priori knowledge of the connection point is required by the model. The discontinuous change in slope $\frac{\partial P}{\partial E}$ is automatically incorporated by the combination of energy-based hysterons or kernels and the densities used to incorporate material and field nonhomogeneities. The automatic enforcement of the 'deletion' property when exiting minor loops is important both for material characterization and the development of model-based compensators for linear feedback control design.

\section{Lognormal/Normal Densities — Identification Using Full Data Set}

Using the full set of data from the 7 loops, we also estimated the parameters $\eta, c, b, C$ and $\bar{E}_{c}$ in the discretized model (17) in which $\nu_{1}$ and $\nu_{2}$ are specified as the lognormal density (13)

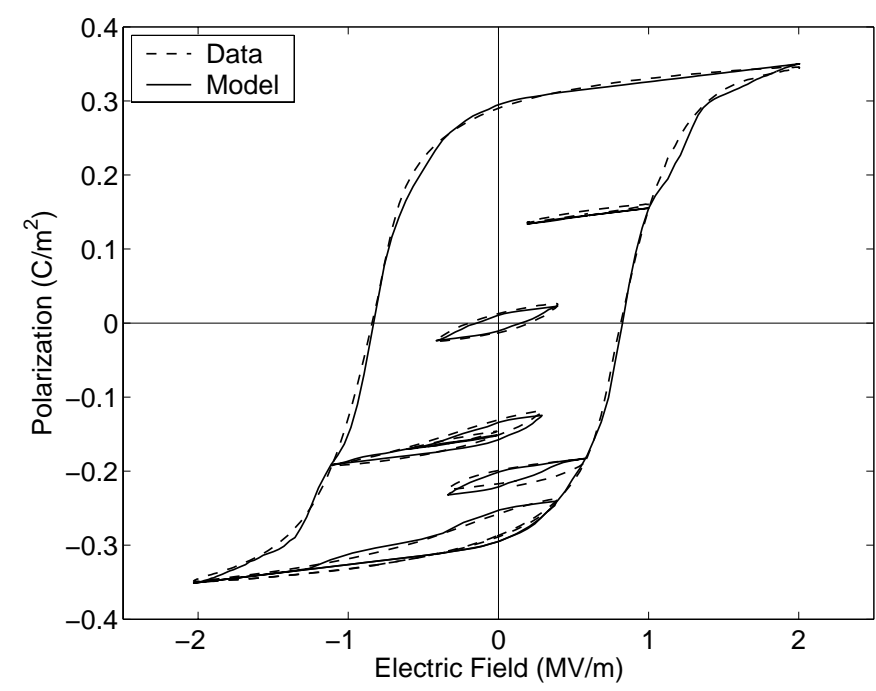

Figure 9: PZT5H data and model with general densities $\nu_{1}$ and $\nu_{2}$ estimated through a fit to full data set. 


\begin{tabular}{l|ccc} 
& (i) & (ii) & (iii) \\
\hline General Densities $\nu_{1}, \nu_{2}\left(N_{i}=N_{j}=80\right)$ & 0.0057 & 0.0115 & 0.0050 \\
Lognormal/Normal Densities $\nu_{1}, \nu_{2}$ & 0.0132 & 0.0136 & 0.0094 \\
General Product Density $\nu\left(N_{i}=N_{j}=24\right)$ & 0.0028 & - & - \\
General Product Density $\nu\left(N_{i}=N_{j}=48\right)$ & 0.0106 & - & - \\
General Product Density $\nu$ - Regularized $\left(N_{i}=N_{j}=24\right)$ & 0.0037 & - & - \\
General Product Density $\nu$ - Regularized $\left(N_{i}=N_{j}=48\right)$ & 0.0024 & - & -
\end{tabular}

Table 2: Residuals $\mathcal{R}$ given by (39) with $\nu_{1}, \nu_{2}$ estimated using the nonlinear constrained minimization algorithm (24) and $\nu$ identified through the quadratic programming algorithm (33) and regularized formulation (38). (i) Identification and residuals over all 7 data sets. (ii) Identification using major symmetric and Rayleigh loops — residual for all 7 data sets. (iii) Identification using major symmetric and Rayleigh loops - residual for major symmetric and Rayleigh loops.

and normal density (15). This yields the parameter values $\eta=8.9 \times 10^{6}, \bar{E}_{c}=7.6 \times 10^{5} \mathrm{~V} / \mathrm{m}$, $c=0.237 \mathrm{~V}^{2} / \mathrm{m}^{2}, b=1.26 \times 10^{5} \mathrm{~V}^{2} / \mathrm{m}^{2}, C=1.4 \times 10^{-12}$ and resulting model fit presented in Figure 11. The corresponding densities are plotted in Figure 12. A comparison between Figure 11 and Figure 9 illustrates a significant loss of accuracy when characterizing the minor loop behavior with this a priori choice of densities. This is quantified by the residual of $\mathcal{R}=0.0132$ which is over twice the residual obtained with general densities.

A comparison between the general densities plotted in Figure 10 and the lognormal/normal densities plotted in Figure 12 indicates the same tendency observed in Section 4.1 for PZT4 the two sets of densities exhibit the same general qualitative behavior but the general densities are significantly less regular than the lognormal and normal functions.

\section{General Densities - Identification Using Symmetric Major and Rayleigh Loop Data}

To illustrate the predictive properties of the model with densities estimated from limiting data, we consider model construction using data from the symmetric major loop and Rayleigh loop. As illustrated in Figure 13, the resulting model fit to these two loops is highly accurate as quantified by the residual $\mathcal{R}=0.0050$ over just these loops. The model predictions for the remaining biased minor loops is less accurate, however, than that obtained when the full data set was used for identification see Figure 9. This loss of accuracy is quantified by the total residual $\mathcal{R}=0.0115$ which, as compiled in Table 2, is over twice that obtained when the full data set was employed for identification.
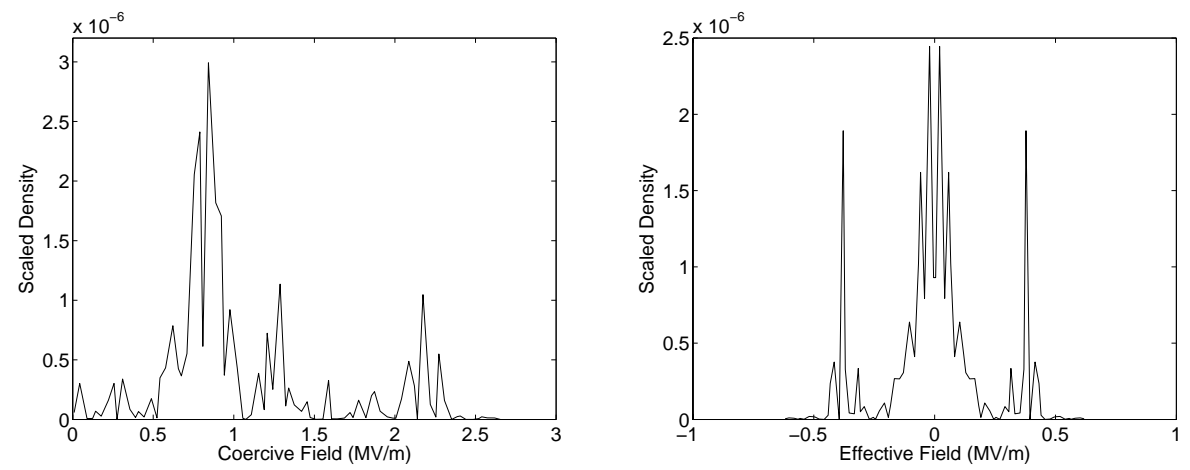

Figure 10: Coercive and effective field densities estimated through a fit to full data set. 


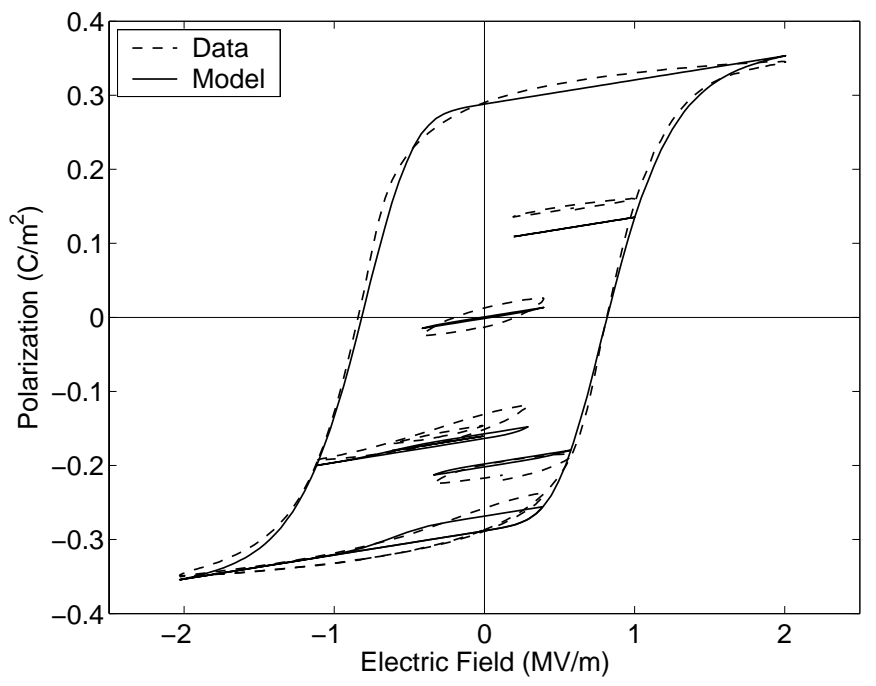

Figure 11: PZT5H data and model prediction with lognormal density $\nu_{1}$ and normal density $\nu_{2}$ having parameters estimated through a fit to full data set.

Finally, we note that because the accuracy of biased minor loops in this regime is highly dependent on the accuracy of the excursion point, even minor discrepancies in the symmetric loop fit can translate into large minor loop errors in regions having a large gradient $\frac{\partial P}{\partial E}$.

\section{Lognormal/Normal Densities — Identification Using Major and Rayleigh Loop Data}

In the final case, a least squares fit to the symmetric major and Rayleigh loop data was used to estimate the parameters $\eta=1.0 \times 10^{7}, \bar{E}_{c}=7.6 \times 10^{5} \mathrm{~V} / \mathrm{m}, c=0.239 \mathrm{~V}^{2} / \mathrm{m}^{2}, b=1.22 \times$ $10^{5} \mathrm{~V}^{2} / \mathrm{m}^{2}, C=1.4 \times 10^{-12}$ in the discretized model (17) constructed using the lognormal and normal densities. The model behavior, plotted in Figure 14, indicates a loss of accuracy in both the fitted loops and the predictions of biased minor loop behavior. This is substantiated by the residual values of 0.0094 over the fitted loops and 0.0136 over the full data set. Hence while the model constructed using the lognormal and normal densities is sufficiently accurate for many applications, a significant improvement in accuracy is provided by the identification of general densities using more comprehensive data sets.
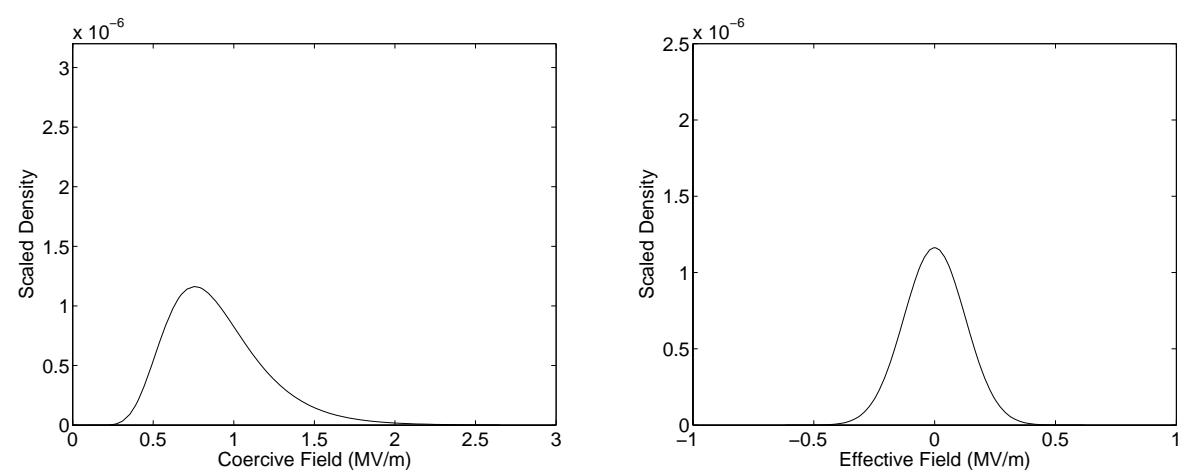

Figure 12: Lognormal coercive and normal effective field densities estimated through a fit to full data set. 


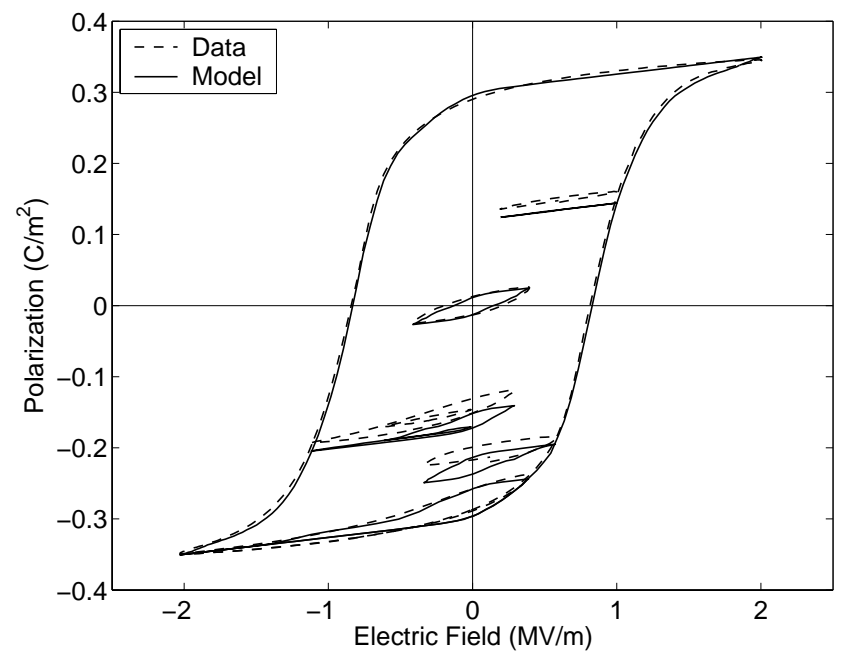

Figure 13: PZT5H data and model predictions with general densities $\nu_{1}$ and $\nu_{2}$ estimated through a fit to symmetric major and Raleigh loop data.

\subsection{PZT5H Characterization — Product Density $\nu$}

In Section 3.3 we summarized a solution technique for estimating the product density $\nu$ based on exploiting the linear parameterization to reformulate the constrained minimization problem as the quadratic programming problem (36). This offers the advantage of providing a well-established solution framework but has the disadvantage of significantly increasing the number of parameters $N_{i} \cdot N_{j}$ versus $N_{i}+N_{j}+1$ - and can be prone to ill-conditioning.

We illustrate in this example the use of this formulation to estimate $\nu$ in (25) using all 7 loops of the PZT5H data originally plotted in Figure 1. To assess the effects of conditioning, we consider two discretization levels $-N_{p}=N_{q}=6 \quad\left(N_{i}=N_{j}=24\right)$ and $N_{p}=N_{q}=12 \quad\left(N_{i}=N_{j}=48\right)-$ both of which are relatively coarse. The model fits and estimated product densities for the two cases are plotted in Figure 15 and 16. As summarized in Table 2, the 7-loop residuals for $N_{i}=N_{j}=24$

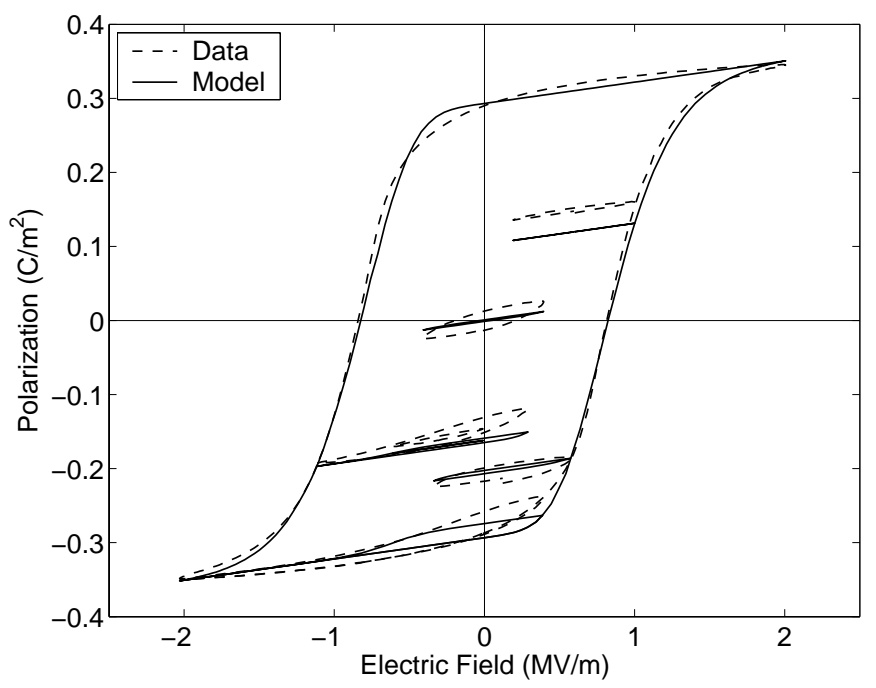

Figure 14: PZT5H data and model prediction with lognormal density $\nu_{1}$ and normal density $\nu_{2}$ having parameters estimated through a fit to symmetric major and Raleigh loop data. 


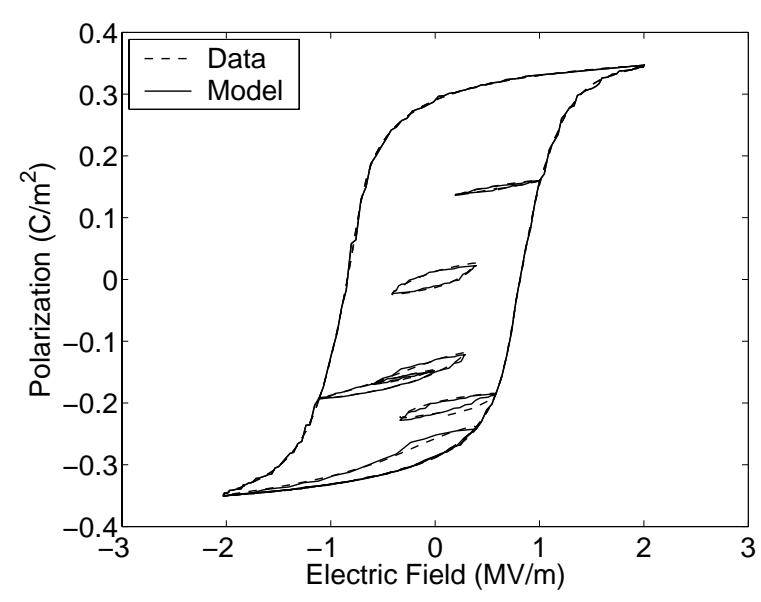

(a)

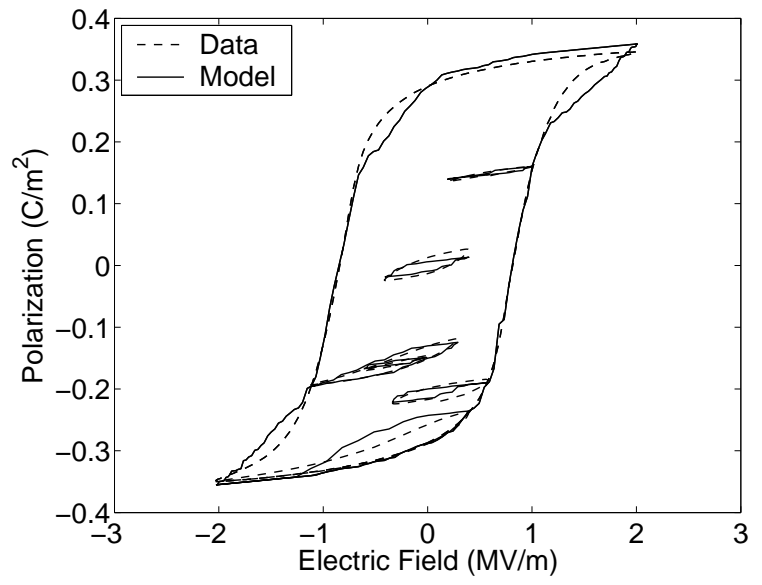

(b)

Figure 15: PZT5H data and model prediction with general product density $\nu$ estimated using the quadratic programming formulation (34) with data from all 7 loops. (a) $N_{i}=N_{j}=24$, and (b) $N_{i}=N_{j}=48$.

and $N_{i}=N_{j}=48$ are respectively 0.0028 and 0.0106 . The fits in Figure 15 in combination with the residual values lead to the following conclusions: (i) the accuracy with the coarse discretization $N_{i}=N_{j}=24$ is comparable to that obtained through solution of the nonlinear constrained optimization problem (24) as illustrated in Figure 9, and (ii) the accuracy degrades significantly as discretization levels are refined. The latter reflects the inherent ill-conditioning associated with the algorithm.

The densities plotted in Figure 16 demonstrate the validity of the decay assumption (iii) in (20) and restriction of the solution operator to compact domains.

Hence while this approach can be highly accurate and relatively efficient for certain discretization levels, care must be taken to avoid degradation in accuracy which can occur as quadrature levels and associated parameter numbers are increased. This motivates consideration of the regularized formulation (38) which is illustrated in Section 4.4.

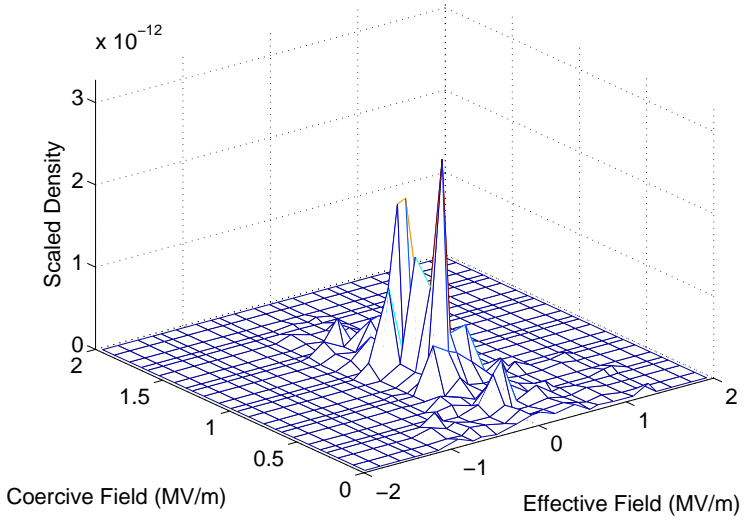

(a)

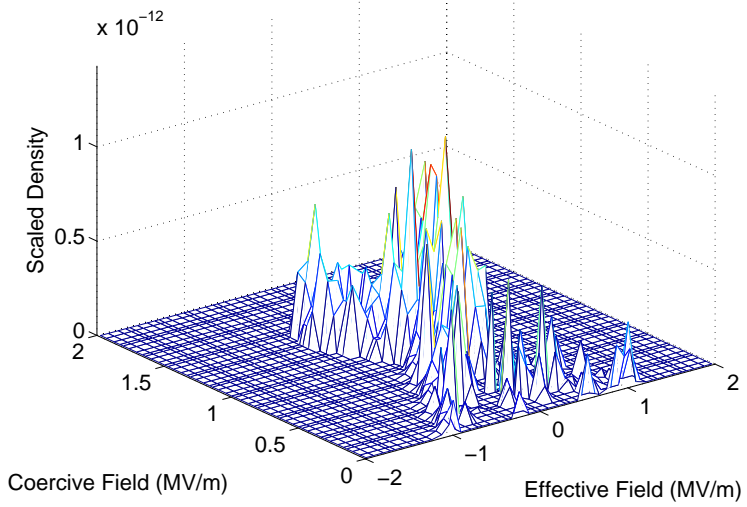

(b)

Figure 16: General product density $\nu$ with (a) $N_{i}=N_{j}=24$, and (b) $N_{i}=N_{j}=48$. 


\subsection{PZT5H Characterization — Product Density $\nu$}

The regularized least squares formulation (38) stabilizes the pseudoinverse associated with the inverse problem by shifting singular values away from the origin. The model fits obtained using (38) with $\alpha=5 \times 10^{20}$ for $N_{i}=N_{j}=24(N=576)$ and $N_{i}=N_{j}=48(N=2304)$, using data from all 7 loops, are illustrated in Figure 17. The corresponding residuals $\mathcal{R}=0.0037$ for $N_{i}=N_{j}=24$ and $\mathcal{R}=0.0024$ for $N_{i}=N_{j}=48$ demonstrate improved accuracy as $N=N_{i} \cdot N_{j}$ is increased which is in contrast with the behavior for the unregularized algorithm. Hence it is observed that regularization in this manner eliminates the instability illustrated in Figure 15 for increasing $N$ and yields highly accurate model representations. Further details illustrating the formulation and effect of regularization techniques for integral hysteresis models are provided in [29].

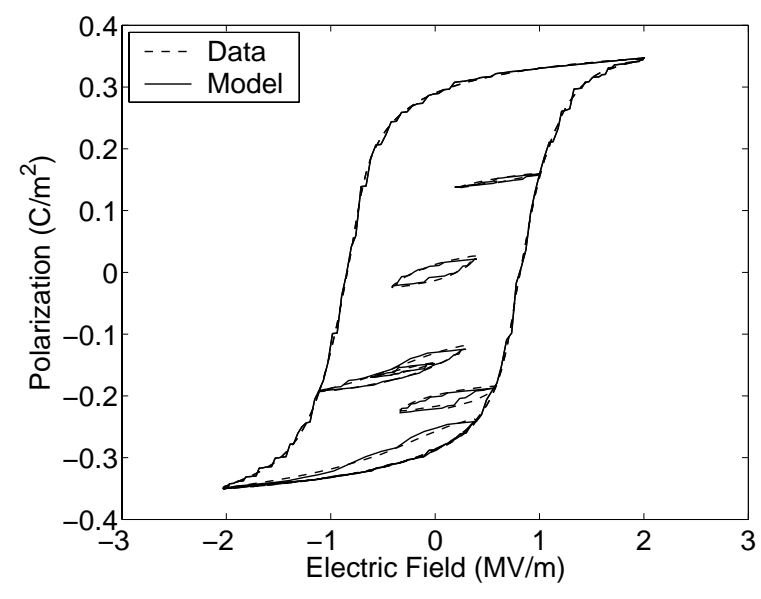

(a)

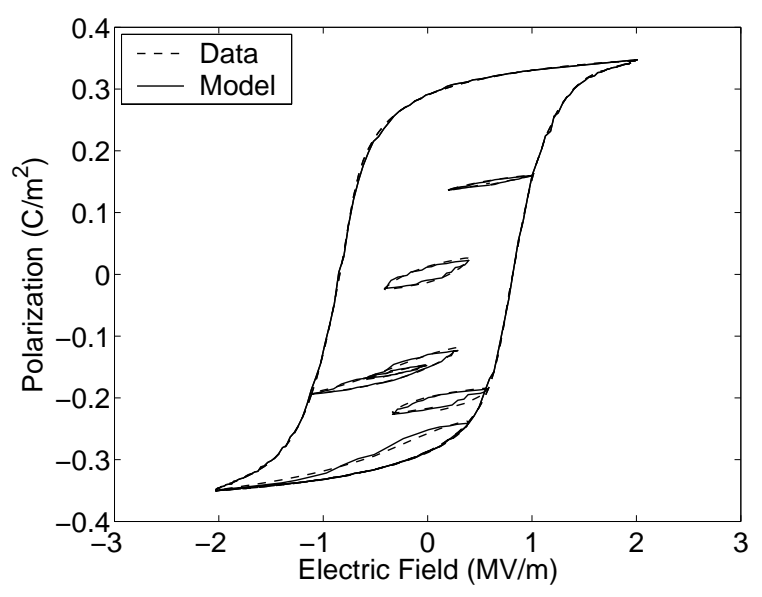

(b)

Figure 17: PZT5H data and model prediction with general product density $\nu$ estimated using Tikhonov regularization with data from all 7 loops. (a) $N_{i}=N_{j}=24$, and (b) $N_{i}=N_{j}=48$.

\section{Concluding Remarks}

In this paper, we have presented a general framework for quantifying hysteresis and constitutive nonlinearities inherent to ferroelectric compounds by combining aspects of the homogenized free energy framework developed in [32] and general Preisach models employing general densities or measures [10, 24, 25, 28]. In the first step of the development, Helmholtz and Gibbs energy relations are constructed at the lattice level. By directly enforcing necessary conditions to minimize energy for regimes in which thermal relaxation mechanisms are negligible, employing Boltzmann theory to balance the relative thermal energy and Gibbs energy, we then construct mesoscale polarization relations. These relations can subsequently be extrapolated to provide suitable macroscopic models for homogeneous, single crystal compounds. For nonhomogeneous, polycrystalline materials with variable effective fields, the local relations provide kernels or hysterons which serve as a basis with material and field variations incorporated through the assumption that local coercive and effective field parameters are manifestations of underlying stochastic distributions rather than constants. In the original development [32], models were based on the a priori assumption of lognormal and normal densities for the coercive and effective fields whereas we employ here general densities which are estimated through fits to experimental data. This provides the models with the flexibility of Preisach models while retaining the advantages associated with the energy formulation for the hysterons. 
Form a theoretical perspective, the models incorporate reversibility and incorporate certain rate and temperature-dependencies directly into the polarization kernel or hysteron. Furthermore, they guarantee closure of biased minor loops in quasistatic or low frequency drive regimes, and ensure the 'deletion' property, but permit accommodation and after-effects. As detailed in [9] in the context of magnetic materials, several of these properties are not inherent to classical Preisach models and require extensions that diminish the efficiency of extended Preisach formulations for transducer design and model-based control design.

The extension of the theory in [32] through formulation in terms of general densities rather than a priori choices of lognormal and normal densities has significant ramifications from the perspective of both material characterization and control design. For material characterization, formulation in terms of general densities provides additional accuracy for certain hard materials and drive regimes involving multiple biased minor loops as illustrated in the context of PZT4 and PZT5H data. For model-based control design, the advantages are even more profound - the model formulated in terms of general densities exhibits a linear dependence on the parameters whereas the model constructed from lognormal and normal densities exhibits a nonlinear dependence on parameters. In the latter case, one is limited to nonlinear adaptive parameter estimation techniques of the type developed in $[14,15,22]$ and there are few options if considering adaptive control design. For the linear parameterization associated with the general density formulation, one can consider a broad range of adaptive identification and control designs $[13,35]$ which extends significantly the flexibility of the method.

It should be noted that whereas the identification of general densities using constrained optimization algorithms can be significantly more expensive than identification of parameters in the lognormal and normal densities, the two formulations are equally efficient to implement once parameters have been identified. Hence both formulations are viable for subsequent use in material characterization, transducer and system design, and model-based control design and implementation.

\section{Acknowledgements}

A.H. was supported by the DARPA subcontract 1000-G-CF980 and the NSF grant CMS-0201560 whereas the research of R.C.S. was supported in part through the NSF grant CMS-0099764 and in part by the Air Force Office of Scientific Research under the grant AFOSR-F49620-01-1-0107. The authors would like to thank Joshua Smith for the numerical characterization of the PZT4 data.

\section{References}

Note: Center for Research in Scientific Computation Technical Reports can be accessed at the web site http://www.ncsu.edu/crsc/reports.html.

[1] J.C. Anderson, Dielectrics, Reinhold Publishing Corporation, New York, 1964.

[2] H.T. Banks, A.J. Kurdila and G. Webb, "Identification of hysteretic control influence operators representing smart actuators Part I: Formulation," Mathematical Problems in Engineering, 3, pp. 287-328, 1997.

[3] H.T. Banks, A.J. Kurdila and G. Webb, "Identification of hysteretic control influence operators representing smart actuators Part II: Convergent approximations," Journal of Intelligent Material Systems and Structures, 8(6), pp. 536-550, 1997. 
[4] W. Chen and C.S. Lynch, "A model for simulating polarization switching and AF-F phase changes in ferroelectric ceramics," Journal of Intelligent Material Systems and Structures, 9, pp. 427-431, 1998.

[5] W. Chen and C.S. Lynch, "A micro-electro-mechanical model for polarization switching of ferroelectric materials," Acta Materialia, 46(15), pp. 5303-5311, 1998.

[6] X. Chen, D.N. Fang and K.C. Hwang, "Micromechanics simulation of ferroelectric polarisation switching," Acta Materialia, 45(8), pp. 3181-3189, 1997.

[7] L.E. Cross, "Relaxor ferroelectrics," Ferroelectrics, 76, pp. 241-267, 1987.

[8] A. Daniele, S. Salapaka, M.V. Salapaka and M. Dahleh, "Piezoelectric scanners for atomic force microscopes: Design of lateral sensors, identification and control," in Proceedings of the American Control Conference, San Diego, CA, pp. 253-257, 1999.

[9] E. Della Torre, Magnetic Hysteresis, IEEE Press, New York, 1999.

[10] P. Ge and M. Jouaneh, "Modeling hysteresis in piezoceramic actuators," Precision Engineering, 17, pp. 211-221, 1995.

[11] C.L. Hom and N. Shankar, "Modeling nonlinearity in electrostrictive sonar transducers," Journal of the Acoustical Society of America, 104(4), pp. 1903-1913, 1998.

[12] L. Huang and H.F. Tiersten, "An analytic description of slow hysteresis in polarized ferroelectric ceramic actuators," Journal of Intelligent Material Systems and Structures, 9, pp. 417-426, 1998.

[13] P.A. Ioannou and J. Sun, Robust Adaptive Control, Prentice-Hall, Upper Saddle River, NJ, 1996.

[14] A. Kojic, C. Cao and A. M. Annaswamy, "Parameter Convergence in systems with Convex/Concave Parameterization," Proceedings of the 2000 American Control Conf., pp. 2240-2244, 2000 .

[15] A-P Loh, A. M. Annaswamy and F. P. Skantze, "Adaptation in the Presence of a General Nonlinear Parameterization: An Error Model Approach," IEEE Transactions on Automatic Control, 44(9), pp. 1634-1652, 1999.

[16] W. Lu, D-N. Fang and K-C. Hwang, "Nonlinear electric-mechanical behavior and micromechanics modelling of ferroelectric domain evolution," Acta Materialia, 47(10), pp. 2913-2926, 1999.

[17] J.A. Main and E. Garcia, "Design impact of piezoelectric actuator nonlinearities," Journal of Guidance, Control, and Dynamics, 20(2), pp. 327-332, 1997.

[18] J.A. Main, D. Newton, L. Massengil and E. Garcia, "Efficient power amplifiers for piezoelectric applications," Smart Materials and Structures, 5(6), pp. 766-775, 1996.

[19] J.E. Miesner and J.P. Teter, "Piezoelectric/magnetostrictive resonant inchworm motor," Proceedings of SPIE, Smart Structures and Materials, Volume 2190, pp. 520-527, 1994.

[20] T. Mitsui, I. Tatsuzaki and E. Nakamura, An Introduction to the Physics of Ferroelectrics, Gordon and Breach Science Publishers, New York, 1976. 
[21] A.J. Moulson and J.M. Herbert, Electroceramic: Materials, Properties, Applications, Chapman and Hill, New York, 1990.

[22] J.M. Nealis and R.C. Smith, "Nonlinear adaptive parameter estimation algorithms for hysteresis models of magnetostrictive actuators," Proceedings of the SPIE, Smart Structures and Materials 2002, Volume 4693, pp. 25-36, 2002.

[23] M. Omura, H. Adachi and Y. Ishibashi, "Simulations of ferroelectric characteristics using a one-dimensional lattice model," Japanese Journal of Applied Physics, 30(9B), pp. 2384-2387, 1991.

[24] F. Preisach, "Uber die magnetische nachwirkung," Zeitschrift fur Physik, 94, pp. 277-302, 1935.

[25] G. Robert, D. Damjanovic and N. Setter, "Preisach modeling of piezoelectric nonlinearity in ferroelectric ceramics," Journal of Applied Physics, 89(9), pp. 5067-5074, 2001.

[26] S. Salapaka, A. Sebastian, J.P. Cleveland and M.V. Salapaka, "High bandwidth nanopositioner: A robust control approach," Review of Scientific Instruments, 73(9), pp. 3232-3241, 2002.

[27] G. Schitter, P. Menold, H.F. Knapp, F. Allgower and A. Stemmer, "High performance feedback for fast scanning atomic force microscopes," Review of Scientific Instruments, 72(8), pp. 3320$3327,2001$.

[28] M.E. Shirley and R. Venkataraman, "On the identification of Preisach measures," Proceedings of the SPIE, Smart Structures and Materials 2003, Modeling, Signal Processing, and Control, Vol. 5049, pp. 326-336, 2003.

[29] R.C. Smith and A.G. Hatch, "Parameter Estimation Techniques for a Polarization Hysteresis Model," CRSC Technical Report CRSC-TR04-19; Proceedings of the SPIE, Smart Structures and Materials 2004, to appear.

[30] R.C. Smith and C.L. Hom, "Domain wall theory for ferroelectric hysteresis," Journal of Intelligent Material Systems and Structures, 10(3), pp. 195-213, 1999.

[31] R.C. Smith and Z. Ounaies, "A domain wall model for hysteresis in piezoelectric materials," Journal of Intelligent Material Systems and Structures, 11(1), pp. 62-79, 2000.

[32] R.C. Smith, S. Seelecke, Z. Ounaies and J. Smith, "A free energy model for hysteresis in ferroelectric materials," Journal of Intelligent Material Systems and Structures, 14(11), 2003, pp. 719-739.

[33] R.C. Smith and S. Seelecke, "An energy formulation for Preisach models," Proceedings of the SPIE, Smart Structures and Materials 2002, Volume 4693, pp. 173-182, 2002.

[34] X. Tan, R. Venkataraman and P.S. Krishnaprasad, "Control of hysteresis: Theory and experimental results," Smart Structures and Materials 2001, Modeling, Signal Processing and Control in Smart Structures, SPIE Vol. 4326, pp. 101-112, 2001.

[35] G. Tao and P. V. Kokotović, Adaptive Control of Systems with Actuator and Sensor Nonlinearities, John Wiley and Sons, New Jersey, 1996.

[36] C.R. Vogel, Computational Methods for Inverse Problems, SIAM, Philadelphia, PA, 2002.

[37] D. Zwillinger, Editor-in-Chief, CRC Standard Mathematical Tables and Formulae, 30th Edition, CRC Press, Boca Raton, 1996. 